TRANSACTIONS OF THE

AMERICAN MATHEMATICAL SOCIETY

Volume 362, Number 6, June 2010, Pages 3069-3094

S 0002-9947(09)04968-X

Article electronically published on December 17, 2009

\title{
POISSON SUSPENSIONS AND ENTROPY FOR INFINITE TRANSFORMATIONS
}

\author{
ÉLISE JANVRESSE, TOM MEYEROVITCH, EMMANUEL ROY \\ AND THIERRY DE LA RUE
}

\begin{abstract}
The Poisson entropy of an infinite-measure-preserving transformation is defined in the 2005 thesis of Roy as the Kolmogorov entropy of its Poisson suspension. In this article, we relate Poisson entropy with other definitions of entropy for infinite transformations: For quasi-finite transformations we prove that Poisson entropy coincides with Krengel's and Parry's entropy. In particular, this implies that for null-recurrent Markov chains, the usual formula for the entropy, $-\sum q_{i} p_{i, j} \log p_{i, j}$, holds for any definitions of entropy. Poisson entropy dominates Parry's entropy in any conservative transformation. We also prove that relative entropy (in the sense of Danilenko and Rudolph) coincides with the relative Poisson entropy. Thus, for any factor of a conservative transformation, difference of the Krengel's entropies equals difference of the Poisson entropies. In case there already exists a factor with zero Poisson entropy, we prove the existence of a maximum (Pinsker) factor with zero Poisson entropy. Together with the preceding results, this answers affirmatively the question raised by Aaronson and Park about existence of a Pinsker factor in the sense of Krengel for quasi-finite transformations.
\end{abstract}

\section{INTRODUCTION}

The basic question considered in this paper is the following: Is there a "natural" entropy theory for infinite-measure-preserving dynamical systems? Both Krengel 11 and Parry 13 defined notions of entropy for measure-preserving transformations, which elegantly generalize Kolmogorov's entropy of a probability-preserving transformation. It is still an open question whether, for any conservative measurepreserving transformation, Parry's definition of entropy coincides with Krengel's. However, there are known sufficient conditions on a system for these two numbers to coincide, and Krengel's entropy dominates Parry's entropy in general.

In the present paper, we relate Parry's and Krengel's definitions of entropy with Poisson entropy, which is the Kolmogorov entropy of the Poisson suspension, an approach previously taken in [16. The main question here is whether Poisson entropy is equal either to Parry's entropy or to Krengel's entropy (or both) for any conservative measure-preserving transformation. We are yet unable to completely solve this question in full generality, but we give many intermediate results. In particular, we show that Poisson entropy dominates Parry's entropy in general (Theorem 5.2) and that equality of all three definitions of entropy holds for many classes of transformations.

Received by the editors March 25, 2008.

2000 Mathematics Subject Classification. Primary 37A05, 37A35, 37A40, 28D20.

(C)2009 American Mathematical Society

Reverts to public domain 28 years from publication 
As a consequence, we obtain an intuitive expression for the entropy of Poissonsuspensions of null-recurrent Markov chains. We thus correct a mistake in [8], where it was claimed that the entropy is always infinite for Poisson suspensions of null-recurrent Markov chains.

We prove that Poisson entropy is equal to Parry's entropy and Krengel's entropy in the following cases: quasi-finite transformations (Theorem 9.1) and rank-one transformations (Proposition 10.1). We also prove that relative entropy (in the sense of [4]) coincides with relative Poisson entropy (Proposition 7.1). We prove that Poisson entropy is a linear functional, just as Krengel's entropy and Parry's entropy.

In Section 10 we give a spectral criterion for zero Poisson entropy, which was previously shown to imply zero Parry entropy. In Section 11, among other results, we prove the following dichotomy for ergodic quasi-finite infinite measure-preserving transformations: either it is remotely infinite or there exists a maximum (Pinsker) factor with zero Poisson, Krengel and Parry entropy. The proof relies on the existence of perfect Poissonian $\sigma$-algebra and illustrates the interest in using Poisson suspensions to derive results in infinite-measure-preserving ergodic theory via the far more developed finite-measure-preserving case. We also state and prove a strong disjointness result in terms of Poisson suspensions.

\section{Poisson Suspensions and Poisson entropy}

The Poisson suspension $\left(X^{*}, \mathcal{B}^{*}, \mu^{*}, T_{*}\right)$ of a standard, $\sigma$-finite invertible measure-preserving transformation $(X, \mathcal{B}, \mu, T)$ is a canonical method of associating a probability-preserving transformation to a $\sigma$-finite-measure-preserving transformation. Informally, it is a system of non-interacting "identical" particles in $X$, each of which propagates according to the transformation $T$, and such that the expected number of particles in a set $A \in \mathcal{B}$ is determined by $\mu(A)$. Poisson suspensions have been studied in mathematical physics as well as in ergodic theory and probabilistic contexts [7, 8, 9, 19, 20] and recently in [16] and [21.

There are various ways to describe a Poisson suspension. Here is one: Let $X^{*}$ denote the space of measures on $X$, and let $\mathcal{B}^{*}$ denote the $\sigma$-algebra generated by the collection of sets

$$
\left\{\left\{\gamma \in X^{*}: \gamma(B) \in[a, b]\right\}: B \in \mathcal{B}, 0 \leq a \leq b \leq \infty\right\}
$$

The probability measure $\mu^{*}$ on $\left(X^{*}, \mathcal{B}^{*}\right)$ is uniquely defined by requiring that measures of disjoint sets be independent and that the measure of each set $A \in \mathcal{B}$ be Poisson distributed with parameter $\mu(A)$ :

$$
\mu^{*}(\gamma(A)=k)=e^{-\mu(A)} \frac{\mu(A)^{k}}{k !} .
$$

Any measure-preserving map $T:(X, \mathcal{B}, \mu) \rightarrow(Y, \mathcal{C}, \nu)$ naturally gives rise to a measure-preserving map $T_{*}:\left(X^{*}, \mathcal{B}^{*}, \mu^{*}\right) \rightarrow\left(Y^{*}, \mathcal{C}^{*}, \nu^{*}\right)$ by $T_{*} \gamma=\gamma \circ T^{-1}$. If $T$ is an endomorphism, the dynamical system $\left(X^{*}, \mathcal{B}^{*}, \mu^{*}, T_{*}\right)$ is the Poisson suspension of $(X, \mathcal{B}, \mu, T)$.

Following [16], the Poisson entropy of an infinite-measure-preserving transformation is defined as the Kolmogorov entropy of the Poisson suspension. This definition gives rise to a new approach for the entropy theory of infinite-measurepreserving transformations. It retains basic properties of Kolmogorov entropy of finite-measure-preserving transformation: If $S$ is a factor of $T$, its Poisson entropy 
is less than the Poisson entropy of $T$ (the Poisson entropy is thus invariant under weak isomorphism) and the Poisson entropy of $T^{n}$ is $|n|$ times the Poisson entropy of $T$. The definition of Poisson entropy generalizes to infinite-measure-preserving amenable group actions.

As proved in [16, the Poisson entropy of a probability-preserving transformation is equal to its Kolmogorov entropy. Theorem 9.1 of this paper generalizes this fact: For any quasi-finite transformation, the Poisson entropy is equal to Parry's entropy and Krengel's entropy (this holds in particular for finite-measure-preserving systems).

We recall that if $(X, \mathcal{B}, \mu, T)$ is conservative, there exists a unique partition of $X$ into $T$-invariant sets $X_{1}$ and $X_{\infty}$, which are the measurable union of finite (resp. infinite) ergodic components of $\mu$. If $\mu\left(X_{1}\right)=0$, then $T$ is said of type $\mathbf{I I}_{\infty}$, and if $\mu\left(X_{\infty}\right)=0$, of type $\mathbf{I I}_{1}$. Only $\mathbf{I I}_{\infty}$ systems will be of interest to us since the $\mathbf{I I}_{1}$ case reduces to the finite measure case. Moreover the possibility to be confronted to periodic behavior inside the $\mathbf{I I}_{1}$ part brings annoying and uninteresting technical difficulties.

A factor of $T$ is a $\sigma$-finite sub- $\sigma$-algebra $\mathcal{F}$ satisfying $T^{-1} \mathcal{F}=\mathcal{F}$. Observe that the trivial $\sigma$-algebra is never a factor of a $\mathbf{I I}_{\infty}$-system. Remark also that, if $T$ is of type $\mathbf{I I}_{\infty}$, then:

- $\mu$ is continuous;

- any factor of $T$ is of type $\mathbf{I I}_{\infty}$;

- any $\sigma$-finite sub- $\sigma$-algebra $\mathcal{A}$ satisfying $T^{-1} \mathcal{A} \subset \mathcal{A}$ has no atom;

- $\left(X^{*}, \mathcal{B}^{*}, \mu^{*}, T_{*}\right)$ is ergodic.

We will require some notation and simple results about Poisson measures. For each $A \in \mathcal{B}$ and $N \in X^{*}$, denote by $N(A): X^{*} \rightarrow \mathbb{N}$ the random variable on the probability space $\left(X^{*}, \mathcal{B}^{*}, \mu^{*}\right)$ which is the (random) measure of the set $A$. If $A$ has finite measure, $N(A)$ is Poisson distributed with parameter $\mu(A)$. If $\mu(A)=\infty$, $N(A)=\infty \mu^{*}$-almost surely. For a finite or countable partition $\alpha$, we will denote by $N(\alpha)=(N(A))_{A \in \alpha}$ the random vector of Poisson random variables corresponding to $\alpha$. By definition of Poisson suspension, the coordinates of $N(\alpha)$ are independent. If $\mathcal{C} \subset \mathcal{B}$ is a $\sigma$-algebra, denote by $\mathcal{C}^{*}:=\sigma(\{N(A): A \in \mathcal{C}\})$ the sub- $\sigma$-algebra of $\mathcal{B}^{*}$ generated by the Poisson random variables of $\mathcal{C}$. For a measurable partition $\alpha$ of $X$, we write $\alpha^{*}:=(\sigma(\alpha))^{*}$, sometimes regarding this as a (not necessarily countable) partition of $X^{*}$.

It is intuitively clear that lack of atoms for a measure implies no "multiplicities" in the Poisson space of this measure. More formally, we have the following standard lemma:

Lemma 2.1. Assuming there are no atoms of positive measure in $(X, \mathcal{B}, \mu), \mu^{*}$ almost surely there are no multiplicities:

$$
\mu^{*}(\{\exists x \in X: N(\{x\}) \geq 2\})=0 .
$$

Lemma 2.2. Let $\alpha, \beta$ be sub- $\sigma$-algebras of $\mathcal{B}$. Then

$$
(\alpha \cap \beta)^{*}=\alpha^{*} \cap \beta^{*} \bmod \mu^{*} .
$$


Proof. We refer to [18 for details about the Fock space structure of $L^{2}\left(\mu^{*}\right)$ and the exponential $\widetilde{\phi}$ of an operator $\phi$ of $L^{2}(\mu)$. For a $\sigma$-algebra $\xi$, let $\pi_{\xi}$ denote the conditional expectation with respect to $\xi$. It is shown in [18] that $\pi_{\alpha^{*}}=\widetilde{\pi_{\alpha}}$ and $\pi_{\beta^{*}}=$ $\widetilde{\pi_{\beta}}$. Set $H=\left\{f \in L^{2}(\mu), \pi_{\alpha} \pi_{\beta} f=f\right\}$ and $K=\left\{g \in L^{2}\left(\mathcal{P}_{\mu}\right), \pi_{\alpha^{*}} \pi_{\beta^{*}} g=g\right\}$. The von Neumann theorem for contractions implies that $\frac{1}{n} \sum_{k=1}^{n}\left(\pi_{\alpha} \pi_{\beta}\right)^{k} \rightarrow \pi_{H}$ in $L^{2}(\mu)$ and $\frac{1}{n} \sum_{k=1}^{n}\left(\pi_{\alpha^{*}} \pi_{\beta^{*}}\right)^{k} \rightarrow \pi_{K}$ in $L^{2}\left(\mathcal{P}_{\mu}\right)$. But $\pi_{\alpha} \pi_{\beta} f=f$ is equivalent to $\pi_{\alpha} f=\pi_{\beta} f=f$. Therefore $\pi_{H}=\pi_{\alpha \cap \beta}$. For the same reason, $\pi_{K}=\pi_{\alpha^{*} \cap \beta^{*}}$. Moreover, $\frac{1}{n} \sum_{k=1}^{n}\left(\pi_{\alpha} \pi_{\beta}\right)^{k}$ tends to $\widetilde{\pi_{\alpha \cap \beta}}=\pi_{(\alpha \cap \beta)^{*}}$. But, for all $n, \frac{1}{n} \sum_{k=1}^{n}\left(\pi_{\alpha} \pi_{\beta}\right)^{k}=$ $\frac{1}{n} \sum_{k=1}^{n}\left(\pi_{\alpha^{*}} \pi_{\beta^{*}}\right)^{k}$. Thus, by uniqueness of the limit, $\pi_{\alpha^{*} \cap \beta^{*}}=\pi_{(\alpha \cap \beta)^{*}}$, that is, $(\alpha \cap \beta)^{*}=\alpha^{*} \cap \beta^{*}$.

In general, the equality $\left(\mathcal{C}_{1} \vee \mathcal{C}_{2}\right)^{*}=\mathcal{C}_{1}^{*} \vee \mathcal{C}_{2}^{*}$ does not hold. This is true however if the intersection of the $\sigma$-algebras is non-atomic. This is the concern of the following lemma (appearing also in [18]):

Lemma 2.3. Let $\alpha, \beta$ and $\mathcal{C}$ be sub- $\sigma$-algebras of $\mathcal{B}$. Assume that $\mathcal{C}$ is $\sigma$-finite and non-atomic. Then

$$
(\mathcal{C} \vee \alpha \vee \beta)^{*}=(\mathcal{C} \vee \alpha)^{*} \vee(\mathcal{C} \vee \beta)^{*} \bmod \mu^{*}
$$

Proof. Obviously $(\mathcal{C} \vee \alpha \vee \beta)^{*} \supset(\mathcal{C} \vee \alpha)^{*} \vee(\mathcal{C} \vee \beta)^{*}$

To complete the proof of this lemma, we must show that for any $A \in \alpha, B \in \beta$ and $C \in \mathcal{C}$, the random variable $N(A \cap B \cap C)$ is measurable with respect to $(\mathcal{C} \vee \alpha)^{*} \vee(\mathcal{C} \vee \beta)^{*}$ up to a $\mu^{*}$-null set. We can find a sequence $\left(\xi_{n}\right)$ of finite $\mathcal{C}$ measurable partitions increasing to $\mathcal{C}$. Assume $C$ has finite measure. Then by Lemma 2.1, for almost every $\gamma \in X^{*}$, we consider the smallest integer $n(\gamma)$ such that for all $E \in \xi_{n(\gamma)}, \gamma(E \cap C)=0$ or 1. We have

$$
N(A \cap B \cap C)=\sum_{k \in \mathbb{N}} 1_{\{n(\gamma)=k\}} \sum_{E \in \xi_{k}} N(A \cap B \cap C \cap E) .
$$

For $E \in \xi_{k}$, set $N^{\prime}(E):=\min (N(A \cap C \cap E), N(B \cap C \cap E))$. Obviously, $N(A \cap B \cap C \cap E) \leq N^{\prime}(E)$. On the other hand, on the set $\{n(\gamma)=k\}, N(E \cap C)$ $=0$ or 1 ; therefore $N^{\prime}(E)=1$ if and only if $N(A \cap B \cap C \cap E)=1$.

Hence, we can write

$$
N(A \cap B \cap C)=\sum_{k \in \mathbb{N}} 1_{\{n(\gamma)=k\}} \sum_{E \in \xi_{k}} N^{\prime}(E) .
$$

But the right-hand side is measurable with respect to $(\mathcal{C} \vee \alpha)^{*} \vee(\mathcal{C} \vee \beta)^{*}$, so the claim is proved when $C$ has finite measure. In the general case, we can write $C$ as the increasing union of finite-measure, $\mathcal{C}$-measurable sets and get the result in the limit.

A lemma of the same flavor, which applies to monotone sequences of $\sigma$-algebras, was proved in [16], using the corresponding projections in $L^{2}(\mu)$ and $L^{2}\left(\mu^{*}\right)$. 
Lemma 2.4. Let $\left\{\mathcal{B}_{n}\right\}_{n \in \mathbb{N}}$ be a sequence of sub- $\sigma$-algebras of $\mathcal{B}$.

(1) If $\left\{\mathcal{B}_{n}\right\}_{n \in \mathbb{N}}$ is an increasing sequence, then $\bigvee_{n \in \mathbb{N}} \mathcal{B}_{n}^{*}=\left(\bigvee_{n \in \mathbb{N}} \mathcal{B}_{n}\right)^{*}$

(2) If $\left\{\mathcal{B}_{n}\right\}_{n \in \mathbb{N}}$ is a decreasing sequence, then $\bigcap_{n \in \mathbb{N}} \mathcal{B}_{n}^{*}=\left(\bigcap_{n \in \mathbb{N}} \mathcal{B}_{n}\right)^{*}$.

The above equalities are modulo null sets.

\section{The Krengel entropy of a COnservative measure-Preserving TRANSFORMATION}

The Krengel entropy of a conservative measure-preserving transformation $(X, \mathcal{B}$, $\mu, T)$ is defined in [11] as

$$
h_{\mathrm{Kr}}(X, \mathcal{B}, \mu, T):=\sup _{A \in \mathcal{F}_{+}} \mu(A) h\left(A, \mathcal{B} \cap A, \mu_{A}, T_{A}\right),
$$

where $\mathcal{F}_{+}$is the collection of sets in $\mathcal{B}$ with finite positive measure, $\mu_{A}$ is the normalized probability measure on $A$ obtained by restricting $\mu$ to $\mathcal{B} \cap A$, and $T_{A}$ : $A \rightarrow A$ is the induced map on $A$. Recall that this map is defined by

$$
T_{A}(x):=T^{\phi_{A}(x)}(x),
$$

where $\phi_{A}(x):=\min \left\{k \geq 1: T^{k}(x) \in A\right\}$ is the first-return-time map associated to $A$. As soon as $T$ is not purely periodic, Krengel proved that

$$
h_{\mathrm{Kr}}(X, \mathcal{B}, \mu, T)=\mu(A) h\left(A, \mathcal{B} \cap A, \mu_{A}, T_{A}\right),
$$

where $A$ is any finite-measure sweep-out set (i.e. a set such that $\bigcup_{n=0}^{\infty} T^{-n} A=X$ ), which always exists when $T$ is of type $\mathbf{I I}_{\infty}$.

The fact that Krengel's entropy extends Kolmogorov's follows from Abramov's formula. The latter states that when $S: \Omega \rightarrow \Omega$ is an ergodic probability-preserving transformation on $(\Omega, \mathcal{F}, p)$, and $A \in \mathcal{B}$, we have

$$
h\left(A, \mathcal{F} \cap A, p(\cdot \mid A), S_{A}\right)=\frac{1}{p(A)} h(\Omega, \mathcal{F}, p, S) .
$$

\section{THE INFORMATION FUNCTION OF A MEASURABLE PARTITION}

Here we describe a generalization of Shannon's information function. This was previously studied by Klimko and Sucheston [10] and Parry [13]: The information function of a measurable partition $\alpha$ is given by

$$
I_{\mu}(\alpha)(x):= \begin{cases}\log \frac{1}{\mu(\alpha(x))} & \text { if } 0<\mu(\alpha(x))<\infty \\ \infty & \text { if } \mu(\alpha(x))=0 \\ 0 & \text { if } \mu(\alpha(x))=\infty .\end{cases}
$$

By $\alpha(x)$ we mean the unique element in $\alpha$ which contains $x$. Similarly, given two partitions $\alpha_{1}$ and $\alpha_{2}$, the conditional information is defined as

$$
I_{\mu}\left(\alpha_{1} \mid \alpha_{2}\right)(x):= \begin{cases}I_{\mu\left(\cdot \mid \alpha_{2}(x)\right)}\left(\alpha_{1}\right)(x) & \text { if } \mu\left(\alpha_{2}(x)\right)<\infty, \\ I_{\mu}\left(\alpha_{1} \vee\left\{\alpha_{2}(x), X \backslash \alpha_{2}(x)\right\}\right)(x) & \text { otherwise. }\end{cases}
$$

Note that the conditional information retains the following property from the finite-measure case (see [10]):

$$
I_{\mu}\left(\bigvee_{0}^{n-1} T^{k} \alpha\right)=I_{\mu}(\alpha) \circ T^{-(n-1)}+\sum_{j=1}^{n-1} I_{\mu}\left(\alpha \mid \bigvee_{1}^{j} T^{k} \alpha\right) \circ T^{j-(n-1)}
$$


In the sequel, we will need the following lemma (see Theorem 2.2 in 13 for a proof):

Lemma 4.1. Let $(\Omega, \mathcal{F}, p)$ be a probability space, $\alpha$ a measurable partition with $H_{\mu}(\alpha)<\infty$, and $\left\{\mathcal{F}_{n}\right\}_{n \in \mathbb{N}}$ an increasing sequence of sub- $\sigma$-algebras such that $\mathcal{F}=$ $\bigvee_{n \geq 1} \mathcal{F}_{n}$. Then

$$
I_{\mu}\left(\alpha \mid \mathcal{F}_{n}\right) \rightarrow I_{\mu}(\alpha \mid \mathcal{F})
$$

in $L_{1}(\Omega, p)$ and p-a.e.

When $\mathcal{C}$ and $\mathcal{D}$ are sub- $\sigma$-algebras corresponding to partitions $\alpha$ and $\beta$, we write $I_{\mu}(\mathcal{C}):=I_{\mu}(\alpha)$ and $I_{\mu}(\mathcal{C} \mid \mathcal{D}):=I_{\mu}(\alpha \mid \beta)$.

Following Parry, if $\mathcal{C}$ and $\mathcal{D}$ are $\sigma$-finite sub- $\sigma$-algebras of $\mathcal{B}$, we define the entropy of $\mathcal{C}$ by

$$
H_{\mu}(\mathcal{C}):=\int_{X} I_{\mu}(\mathcal{C}) d \mu
$$

and the conditional entropy of $\mathcal{C}$ with respect to $\mathcal{D}$ by

$$
H_{\mu}(\mathcal{C} \mid \mathcal{D}):=\int_{X} I_{\mu}(\mathcal{C} \mid \mathcal{D})(x) d \mu(x)
$$

Since $\mathcal{D}$ is $\sigma$-finite, we have $\mu(\beta(x))<\infty$ for $\mu$-almost all $x$ where $\beta$ is the partition associated to $\mathcal{D}$. Hence,

$$
H_{\mu}(\mathcal{C} \mid \mathcal{D})=\int_{X} H_{\mu(\cdot \mid \beta(x))}(\mathcal{C}) d \mu(x)
$$

Finite conditional entropy implies that $\mu$-almost every atom of $\mathcal{D}$ intersects at most countably many atoms of $\mathcal{C}$.

The following lemma is useful for entropy estimates of a Poisson measure.

Lemma 4.2. Assume that $(X, \mathcal{B}, \mu)$ is a Lebesgue space where $\mu$ is continuous and infinite. Let $\mathcal{D} \subset \mathcal{C}$ be $\sigma$-finite sub- $\sigma$-algebras of $\mathcal{B}$ with no atom of positive measure. Then

$$
H_{\mu^{*}}\left(\mathcal{C}^{*} \mid \mathcal{D}^{*}\right)=H_{\mu}(\mathcal{C} \mid \mathcal{D})
$$

Proof. Note first that we can take $\mathcal{C}=\mathcal{B}$, and by disintegrating $\mu$ with respect to $\mathcal{D}$ and using the fact that $(X, \mathcal{B}, \mu)$ is a Lebesgue space with a continuous infinite measure, we can represent $(X, \mathcal{B}, \mu)$ as $(\mathbb{R} \times Y, \mathcal{A} \otimes \mathcal{Y}, \mu)$, where $\mathcal{A}$ is the Borel $\sigma$ algebra and $\mu\left(A_{1} \times A_{2}\right)=\int_{A_{1}} m_{x}\left(A_{2}\right) \lambda(d x), \lambda$ being the Lebesgue measure. Thus $\left(X^{*}, \mathcal{B}^{*}, \mu^{*}\right)$ takes the form $\left((\mathbb{R} \times Y)^{*},(\mathcal{A} \otimes \mathcal{Y})^{*}, \mu^{*}\right)$. This latter Poisson measure has the form of a so-called marked Poisson process (see [3]), namely, we can identify it with $\left(\mathbb{R}^{*} \times Y^{\mathbb{Z}}, \mathcal{A}^{*} \otimes \mathcal{Y}^{\otimes \mathbb{Z}}, \mathbb{P}\right)$ through the mapping $\nu \in(\mathbb{R} \times Y)^{*} \mapsto\left(\gamma,\left\{y_{i}\right\}_{i \in \mathbb{Z}}\right)$, where

- $\gamma$ is the projection of $\nu$ on $\mathbb{R}: \gamma=\sum_{i \in \mathbb{Z}} \delta_{t_{i}(\gamma)}$, with

$$
\cdots<t_{-n}(\gamma)<\cdots<t_{-1}(\gamma)<t_{0}(\gamma) \leq 0<t_{1}(\gamma)<\cdots<t_{n}(\gamma)<\cdots,
$$

- $\left(y_{i}\right)_{i \in \mathbb{Z}}$ are defined by $\nu=\sum_{i \in \mathbb{Z}} \delta_{\left(t_{i}(\gamma), y_{i}\right)}$. 
Here $\mathbb{P}\left(C_{1} \times C_{2}\right)=\int_{C_{1}} p_{\gamma}\left(C_{2}\right) \lambda^{*}(d \gamma)$ with $p_{\gamma}=\bigotimes_{i \in \mathbb{Z}} m_{t_{i}(\gamma)}$. The verification of this fact amounts to evaluating the Laplace transform for a positive function $f$ on $\mathbb{R} \times Y$ once we have recalled that the Laplace transform of a Poisson measure on a space $(\Omega, \mathcal{F}, m)$ is

$$
\int_{\Omega^{*}} \exp \left(-\int_{\Omega} g(\omega) \nu(d \omega)\right) m^{*}(d \nu)=\exp \int_{\Omega}(\exp (-g(\omega))-1) m(d \omega)
$$

for a non-negative measurable function $g$ on $(\Omega, \mathcal{F})$. So let $f$ be a non-negative measurable function on $\mathbb{R} \times Y$ :

$$
\begin{aligned}
& \int_{\mathbb{R}^{*}}\left(\int_{Y^{\mathbb{Z}}} \exp \left(-\sum_{i \in \mathbb{Z}} f\left(t_{i}(\gamma), y_{i}\right)\right) \otimes_{i \in \mathbb{Z}} m_{t_{i}(\gamma)}\left(d\left\{y_{i}\right\}_{i \in \mathbb{Z}}\right)\right) \lambda^{*}(d \gamma) \\
& =\int_{\mathbb{R}^{*}} \exp \left\{\sum_{i \in \mathbb{Z}} \log \left(\int_{Y} \exp \left(-f\left(t_{i}(\gamma), y\right)\right) m_{t_{i}(\gamma)}(d y)\right)\right\} \lambda^{*}(d \gamma) \\
& =\int_{\mathbb{R}^{*}} \exp \left\{\int_{\mathbb{R}} \log \left(\int_{Y} \exp (-f(t, y)) m_{t}(d y)\right) d \gamma(t)\right\} \lambda^{*}(d \gamma) \\
& \left.=\exp \int_{\mathbb{R}}\left\{\exp \left(\log \left(\int_{Y} \exp (-f(t, y)) m_{t}(d y)\right)\right)-1\right\} d t \text { thanks to (4) }\right) \\
& =\exp \int_{\mathbb{R}}\left(\int_{Y}(\exp (-f(t, y))-1) m_{t}(d y)\right) d t \\
& =\exp \int_{\mathbb{R} \times Y}(\exp (-f(z))-1) \mu(d z),
\end{aligned}
$$

which is the Laplace transform of the Poisson measure of distribution $\mu^{*}$ evaluated at $f$, thanks again to (4).

In this setting, we can rewrite $H_{\mu^{*}}\left(\mathcal{C}^{*} \mid \mathcal{D}^{*}\right)$ as

$$
\int_{\mathbb{R}^{*}} d \lambda^{*}(\gamma) H_{p_{\gamma}}\left(\mathcal{Y}^{\otimes \mathbb{Z}}\right)=\int_{\mathbb{R}^{*}} d \lambda^{*}(\gamma) \sum_{i \in \mathbb{Z}} H_{m_{t_{i}}}(\mathcal{Y})=\int_{\mathbb{R}^{*}} d \lambda^{*}(\gamma) \int_{\mathbb{R}} d \gamma(t) H_{m_{t}}(\mathcal{Y}),
$$

which is equal to

$$
\int_{\mathbb{R}} d \lambda(t) H_{m_{t}}(\mathcal{Y})=H_{\mu}(\mathcal{C} \mid \mathcal{D})
$$

since $\lambda=\int \gamma d \lambda^{*}(\gamma)$.

\section{PARRY'S ENTROPY}

In this section we recall Parry's definition of entropy for a measure-preserving transformation and prove that Parry's entropy is dominated by Poisson entropy.

Parry [13] defines the entropy of a measure-preserving transformation by

$$
h_{\mathrm{Pa}}(X, \mathcal{B}, \mu, T):=\sup _{T^{-1} \mathcal{C} \subset \mathcal{C}} H_{\mu}\left(\mathcal{C} \mid T^{-1} \mathcal{C}\right),
$$

where the supremum is taken over all $\sigma$-finite sub- $\sigma$-algebras $\mathcal{C}$ of $\mathcal{B}$ such that $T^{-1} \mathcal{C} \subset \mathcal{C}$. For probability-preserving transformations, this definition coincides with the standard definition of Kolmogorov's entropy.

The following theorem was proved by Parry (Theorem 10.11 in [13]). 
Theorem 5.1. Let $(X, \mathcal{B}, \mu, T)$ be a measure-preserving conservative transformation. Then

$$
h_{P a}(X, \mathcal{B}, \mu, T) \leq h_{K r}(X, \mathcal{B}, \mu, T) .
$$

Replacing Krengel entropy by Poisson entropy, we prove a similar result:

Theorem 5.2. Let $(X, \mathcal{B}, \mu, T)$ be a $\mathbf{I I}_{\infty}$ transformation. Then

$$
h_{P a}(X, \mathcal{B}, \mu, T) \leq h\left(X^{*}, \mathcal{B}^{*}, \mu^{*}, T_{*}\right) .
$$

Proof. Let $\mathcal{C} \subset \mathcal{B}$ be a sub-invariant $\sigma$-finite sub- $\sigma$-algebra; that is, $T^{-1} \mathcal{C} \subset \mathcal{C}$. Since $\mathcal{B}$ has no atom of positive $\mu$-measure and $\mathcal{C}$ is $\sigma$-finite, the same follows for $\mathcal{C}$, and so by Lemma 4.2 we know that

$$
H_{\mu}\left(\mathcal{C} \mid T^{-1} \mathcal{C}\right)=H_{\mu^{*}}\left(\mathcal{C}^{*} \mid T_{*}^{-1} \mathcal{C}^{*}\right) .
$$

Now it follows that

$$
\sup _{T^{-1} \mathcal{C} \subset \mathcal{C}} H_{\mu}\left(\mathcal{C} \mid T^{-1} \mathcal{C}\right) \leq \sup _{T_{*}^{-1} \mathcal{D} \subset \mathcal{D}} H_{\mu^{*}}\left(\mathcal{D} \mid T^{-1} \mathcal{D}\right),
$$

where the supremum on the right-hand side is over all factors $\mathcal{D} \subset \mathcal{B}^{*}$, which proves the theorem.

\section{An upper Bound FOR the POISSON ENTROPY}

Whenever the measure-preserving system $(X, \mathcal{B}, \mu, T)$ is implicitly clear from the context, for any measurable partition $\alpha$ of $X$ and $-\infty \leq i<j \leq+\infty$, we write $\alpha_{i}^{j}:=\bigvee_{k=i}^{j} T^{k} \alpha$. We will assume from now on that $T$ is an automorphism; that is, $T^{-1} \mathcal{B}=\mathcal{B}$ with equality modulo $\mu$. Also, we write $\widehat{\alpha}=\alpha_{-\infty}^{\infty}$.

Recall that $\mathcal{F}_{+}$denotes the collection of sets in $\mathcal{B}$ with finite positive measure. We say that a countable partition $\alpha$ of $X$ is local with core $A \in \mathcal{F}_{+}$if

$$
A^{c} \in \alpha \quad \text { and } \quad H_{\mu}(\alpha)<\infty .
$$

In other words, $\alpha$ is a finite-entropy partition of a set $A$ of finite measure, to which the complement of $A$ is added. Note that $H_{\mu}(\alpha)<\infty$ implies $H_{\mu^{*}}\left(\alpha^{*}\right)<\infty$. Since a partition with finite entropy is always at most essentially countable, we see in particular that $\alpha^{*}$ is a countable partition of $X^{*}$ modulo $\mu^{*}$. In [2] and [10] co-finite partitions were considered. With our terminology, these are finite, local partitions.

For a partition $\alpha$ of $X$, define

$$
\hat{h}(X, \mu, T, \alpha):=\liminf _{n \rightarrow \infty} \frac{1}{n} H_{\mu}\left(\alpha_{0}^{n-1}\right) .
$$

In the case where $\mu$ is a probability measure and $H_{\mu}(\alpha)<\infty$, this is equal to the Kolmogorov entropy of the factor generated by $\left\{T^{-n} \alpha\right\}_{n=0}^{\infty}$.

Lemma 6.1. Let $(X, \mathcal{B}, \mu, T)$ be a $\mathbf{I I}_{\infty}$ transformation and let $\alpha$ be a local partition whose core $A$ is a sweep-out set. Then we have

$$
\lim _{n \rightarrow \infty} \sup \left\{\mu(a): a \in \bigvee_{k=0}^{n-1} T^{-k} \alpha \cap \mathcal{F}_{+}\right\}=0 .
$$


Proof. Since $A$ is a sweep-out set, the first-return-time map $\phi_{A}$ is finite almost everywhere, and this easily implies that the same is true for the first-return-time $\operatorname{map} \psi_{A}(x)=\min \left\{n>0: T^{-n}(x) \in A\right\}$.

Choose $k \in \mathbb{N}$ so large that $\mu\left(A \cap\left\{\phi_{A}(x)>k\right\}\right)<\epsilon$ and $\mu\left(A \cap\left\{\psi_{A}(x)>k\right\}\right)<\epsilon$. For all $n \geq 1$, let

$$
B_{n}:=B \cap T_{A}^{-1} B \cap \cdots \cap T_{A}^{-n} B
$$

where

$$
B:=\left\{x \in A: \phi_{A}(x)<k\right\} .
$$

We claim that $\lim _{n \rightarrow \infty} \mu\left(B_{n}\right)=0$. Indeed, if $\lim _{n \rightarrow \infty} \mu\left(B_{n}\right)>0$, then $B_{\infty}:=$ $\bigcap_{n \geq 1} B_{n}$ is a set of positive measure, is $T_{A}$-invariant, and its first-return-time map $\phi_{B_{\infty}}$ is bounded by $k$. Then $B_{\infty} \cup T B_{\infty} \cup \cdots \cup T^{k} B_{\infty}$ is a set of finite positive measure which is $T$-invariant, which contradicts the hypothesis that $(X, \mathcal{B}, \mu, T)$ is II $_{\infty}$.

Consider $a \in \bigvee_{j=0}^{k n} T^{-j} \alpha \cap \mathcal{F}_{+}$, and let $0 \leq j_{1}<\cdots<j_{\ell} \leq k n$ be the corresponding passages in $A$ : If $j_{1}>k$, then $a \subset T^{-j_{1}}\left[A \cap\left\{\psi_{A}>k\right\}\right]$. If there exists $i$ such that $j_{i+1}-j_{i}>k$ or if $k n-j_{\ell}>k$, then $a$ is contained in $T^{-j}\left[A \cap\left\{\phi_{A}>k\right\}\right]$ for some $j$. Otherwise, $a$ is contained in $T^{-j} B_{n}$ for some $j$. If $n$ is large enough, we get that $\mu(a)<\epsilon$, which concludes the proof.

Proposition 6.2. Let $(X, \mathcal{B}, \mu, T)$ be a $\mathbf{I I}_{\infty}$ transformation and let $\alpha$ be a local partition, whose core is a sweep-out set. We have

$$
h\left(X^{*},(\hat{\alpha})^{*}, \mu^{*}, T_{*}\right) \leq \hat{h}(X, \mu, T, \alpha) .
$$

Proof. For any $n \geq 1$ and any $p \geq 1$, since $\left(\left(\alpha_{0}^{p}\right)^{*}\right)_{0}^{n-1} \prec\left(\alpha_{0}^{n-1+p}\right)^{*}$, we have

$$
\frac{1}{n} H_{\mu^{*}}\left(\left(\left(\alpha_{0}^{p}\right)^{*}\right)_{0}^{n-1}\right) \leq \frac{n+p}{n} \frac{1}{n+p} H_{\mu^{*}}\left(\left(\alpha_{0}^{n-1+p}\right)^{*}\right) .
$$

But $H_{\mu^{*}}\left(\left(\alpha_{0}^{n-1+p}\right)^{*}\right)=\sum_{a \in \alpha_{0}^{n-1+p}} f(\mu(a))$, where $f(x)$ is the entropy of a Poisson random variable with parameter $x$. An easy computation shows that $f(\epsilon) \sim-\epsilon \log \epsilon$ at the origin. By Lemma 6.1. $\sup _{a \in \alpha_{0}^{n-1+p} \cap \mathcal{F}_{+}} \mu(a)$ tends to 0 as $n$ tends to infinity. Hence we easily deduce that $H_{\mu^{*}}\left(\left(\alpha_{0}^{n-1+p}\right)^{*}\right) \sim H_{\mu}\left(\alpha_{0}^{n-1+p}\right)$ as $n$ tends to infinity, and we get

$$
\lim _{n \rightarrow \infty} \frac{1}{n} H_{\mu^{*}}\left(\left(\left(\alpha_{0}^{p}\right)^{*}\right)_{0}^{n-1}\right) \leq \liminf _{n \rightarrow \infty} \frac{n+p}{n} \frac{1}{n+p} H_{\mu}\left(\alpha_{0}^{n-1+p}\right)=\hat{h}(X, \mu, T, \alpha) .
$$

Taking the limit in $p$, we obtain the desired inequality.

\section{Relative Poisson entropy}

Here $\mathcal{C}$ is an invertible factor $\left(T^{-1} \mathcal{C}=\mathcal{C}\right)$ of $(X, \mathcal{B}, \mu, T)$. The relative entropy of $T$ with respect to $\mathcal{C}$ is defined by

$$
h(X, \mathcal{B}, \mu, T \mid \mathcal{C}):=\sup _{\alpha} \lim _{n \rightarrow \infty} \frac{1}{n} H_{\mu}\left(\bigvee_{k=0}^{n-1} T^{-k} \alpha \mid \mathcal{C}\right)
$$

where the supremum is taken over all countable partitions $\alpha$ with $H_{\mu}(\alpha \mid \mathcal{C})<\infty$. 
This definition of relative entropy, which is classical for probability-preserving transformations, was applied to $\sigma$-finite measure-preserving actions of countable amenable groups by Danilenko and Rudolph 4 .

Proposition 7.1. Let $\mathcal{C}$ be an invertible factor of a $\mathbf{I I}_{\infty}$ system $(X, \mathcal{B}, \mu, T)$. Then the following quantities are equal:

- $h(X, \mathcal{B}, \mu, T \mid \mathcal{C})$.

- $\lim _{p \rightarrow \infty} \lim _{n \rightarrow \infty} \frac{1}{n} H_{\mu}\left(\bigvee_{k=0}^{n-1} T^{-k} \alpha_{p} \mid \mathcal{C}\right)$, where $\alpha_{p} \uparrow \mathcal{B}$ are a sequence of local partitions with a core $A \in \mathcal{C}$ which is a sweep out set and $H_{\mu}\left(\alpha_{p} \mid \mathcal{C}\right)<\infty$.

- $\sup _{\mathcal{D} \subset \mathcal{B}, T^{-1} \mathcal{D} \subset \mathcal{D}} H_{\mu}\left(\mathcal{D} \mid T^{-1} \mathcal{D} \vee \mathcal{C}\right)(\mathcal{D} \sigma$-finite $)$ - $\sup _{\mathcal{D} \subset \mathcal{B}, T_{*}^{-1} \mathcal{D}^{*} \subset \mathcal{D}^{*}} H_{\mu}\left(\mathcal{D}^{*} \mid T_{*}^{-1} \mathcal{D}^{*} \vee \mathcal{C}^{*}\right)(\mathcal{D} \sigma$-finite $)$.

- $h\left(X^{*}, \mathcal{B}^{*}, \mu^{*}, T_{*} \mid \mathcal{C}^{*}\right)$.

- $\mu(A) h\left(A, \mathcal{B} \cap A, \mu_{A}, T_{A} \mid \mathcal{C} \cap A\right)$ for any sweep-out set $A \in \mathcal{C}$.

Proof. Let $\alpha$ be a local partition whose core $A \in \mathcal{C}$ is a sweep-out set, and such that $H_{\mu}(\alpha \mid \mathcal{C})<\infty$. Then

$$
\begin{aligned}
& H_{\mu}\left(\bigvee_{k=0}^{n-1} T^{-k} \alpha \mid \mathcal{C}\right)=\sum_{k=0}^{n-1} H_{\mu}\left(T^{-k} \alpha \mid \bigvee_{j=k+1}^{n-1} T^{-j} \alpha \vee \mathcal{C}\right) \\
& =\sum_{k=0}^{n-1} H_{\mu}\left(\alpha \mid \bigvee_{j=1}^{n-1-k} T^{-j} \alpha \vee \mathcal{C}\right)=\sum_{k=0}^{n-1} H_{\mu}\left(\alpha \mid \bigvee_{j=1}^{k} T^{-j} \alpha \vee \mathcal{C}\right)
\end{aligned}
$$

Since $H_{\mu}\left(\alpha \mid \bigvee_{j=1}^{k} T^{-j} \alpha \vee \mathcal{C}\right)$ tends to

$$
H_{\mu}\left(\alpha \mid \bigvee_{j=1}^{\infty} T^{-j} \alpha \vee \mathcal{C}\right)=H_{\mu}\left(\bigvee_{j=0}^{\infty} T^{-j} \alpha \mid \bigvee_{j=1}^{\infty} T^{-j} \alpha \vee \mathcal{C}\right)
$$

Cesaro averages give

$$
\lim _{n \rightarrow \infty} \frac{1}{n} H_{\mu}\left(\bigvee_{k=0}^{n-1} T^{-k} \alpha \mid \mathcal{C}\right)=H_{\mu}\left(\bigvee_{j=0}^{\infty} T^{-j} \alpha \mid \bigvee_{j=1}^{\infty} T^{-j} \alpha \vee \mathcal{C}\right)
$$


Now remark that, since $A \in \mathcal{C}, I_{\mu}\left(\alpha \mid \bigvee_{j=1}^{\infty} T^{-j} \alpha \vee \mathcal{C}\right)$ vanishes outside $A$ and $\left(\bigvee_{j=1}^{\infty} T^{-j} \alpha \vee \mathcal{C}\right) \cap A=\left(\bigvee_{j=1}^{\infty} T_{A}^{-j} \alpha \vee \mathcal{C}\right) \cap A$. Thus

$$
\begin{aligned}
H_{\mu}\left(\alpha \mid \bigvee_{j=1}^{\infty} T^{-j} \alpha \vee \mathcal{C}\right) & =\int_{X} I_{\mu}\left(\alpha \mid \bigvee_{j=1}^{\infty} T^{-j} \alpha \vee \mathcal{C}\right) d \mu \\
& =\mu(A) \int_{A} I_{\mu_{A}}\left(\alpha \mid\left(\bigvee_{j=1}^{\infty} T_{A}^{-j} \alpha \vee \mathcal{C}\right) \cap A\right) d \mu_{A} \\
& =\mu(A) H_{\mu_{A}}\left(\alpha \mid\left(\bigvee_{j=1}^{\infty} T_{A}^{-j} \alpha \vee \mathcal{C}\right) \cap A\right) .
\end{aligned}
$$

On the one hand, $\sup _{\alpha} H_{\mu_{A}}\left(\alpha \mid\left(\bigvee_{j=1}^{\infty} T_{A}^{-j} \alpha \vee \mathcal{C}\right) \cap A\right)$ over countable partition of $A$ such that $H_{\mu}(\alpha \mid \mathcal{C})=H_{\mu_{A}}(\alpha \mid \mathcal{C} \cap A)<\infty$ equals $h\left(A, \mathcal{B} \cap A, \mu_{A}, T_{A} \mid \mathcal{C} \cap A\right)$. On the other hand, $H_{\mu}\left(\alpha \mid \bigvee_{j=1}^{\infty} T^{-j} \alpha \vee \mathcal{C}\right) \leq \sup _{\mathcal{D} \subset \mathcal{B}, T^{-1} \mathcal{D} \subset \mathcal{D}} H_{\mu}\left(\mathcal{D} \mid T^{-1} \mathcal{D} \vee \mathcal{C}\right)$. Therefore,

$$
\begin{aligned}
\mu(A) h\left(A, \mathcal{B} \cap A, \mu_{A}, T_{A} \mid \mathcal{C} \cap A\right) & \leq h(X, \mathcal{B}, \mu, T \mid \mathcal{C}) \\
& \leq \sup _{\mathcal{D} \subset \mathcal{B}, T^{-1} \mathcal{D} \subset \mathcal{D}} H_{\mu}\left(\mathcal{D} \mid T^{-1} \mathcal{D} \vee \mathcal{C}\right)
\end{aligned}
$$

Now, since $T$ is of type $\mathbf{I I}_{\infty}$, observe that if $\mathcal{D}$ is a sub-invariant $\sigma$-finite sub- $\sigma$ algebra of $\mathcal{B}$, then $T^{-1} \mathcal{D}$ is non-atomic. We thus have

$$
\begin{aligned}
& H_{\mu}\left(\mathcal{D} \mid T^{-1} \mathcal{D} \vee \mathcal{C}\right) \\
& =H_{\mu}\left(\mathcal{D} \vee T^{-1} \mathcal{D} \vee \mathcal{C} \mid T^{-1} \mathcal{D} \vee \mathcal{C}\right) \\
& =H_{\mu^{*}}\left(\left(\mathcal{D} \vee T^{-1} \mathcal{D} \vee \mathcal{C}\right)^{*} \mid\left(T^{-1} \mathcal{D} \vee \mathcal{C}\right)^{*}\right) \quad \text { by Lemma } 4.2 \\
& =H_{\mu^{*}}\left(\mathcal{D}^{*} \vee\left(T^{-1} \mathcal{D} \vee \mathcal{C}\right)^{*} \mid\left(T^{-1} \mathcal{D} \vee \mathcal{C}\right)^{*}\right) \quad \text { by Lemma } 2.3 \\
& =H_{\mu^{*}}\left(\mathcal{D}^{*} \mid\left(T^{-1} \mathcal{D} \vee \mathcal{C}\right)^{*}\right) \\
& \leq H_{\mu^{*}}\left(\mathcal{D}^{*} \mid T_{*}^{-1} \mathcal{D}^{*} \vee \mathcal{C}^{*}\right) \text {. }
\end{aligned}
$$

Hence,

$$
\begin{aligned}
& \quad \sup _{\mathcal{D} \subset \mathcal{B}, T^{-1} \mathcal{D} \subset \mathcal{D}} H_{\mu}\left(\mathcal{D} \mid T^{-1} \mathcal{D} \vee \mathcal{C}\right) \\
& \quad \leq \sup _{\mathcal{D} \subset \mathcal{B}, T_{*}^{-1} \mathcal{D}^{*} \subset \mathcal{D}^{*}} H_{\mu}\left(\mathcal{D}^{*} \mid T_{*}^{-1} \mathcal{D}^{*} \vee \mathcal{C}^{*}\right) \leq h\left(X^{*}, \mathcal{B}^{*}, \mu^{*}, T_{*} \mid \mathcal{C}^{*}\right) .
\end{aligned}
$$


Moreover, by taking an increasing sequence $\alpha_{p}$ of countable partitions with core $A$,

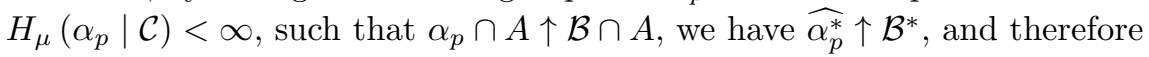

$$
h\left(X^{*}, \mathcal{B}^{*}, \mu^{*}, T_{*} \mid \mathcal{C}^{*}\right)=\lim _{p \rightarrow \infty n \rightarrow \infty} \lim _{n} \frac{1}{n} H_{\mu^{*}}\left(\bigvee_{k=0}^{n-1} T_{*}^{-k} \alpha_{p}^{*} \mid \mathcal{C}^{*}\right) .
$$

But

$$
\begin{aligned}
& \frac{1}{n} H_{\mu^{*}}\left(\bigvee_{k=0}^{n-1} T_{*}^{-k} \alpha_{p}^{*} \mid \mathcal{C}^{*}\right) \leq \frac{1}{n} H_{\mu^{*}}\left(\left(\bigvee_{k=0}^{n-1} T^{-k} \alpha_{p}\right)^{*} \vee \mathcal{C}^{*} \mid \mathcal{C}^{*}\right) \\
& \leq \frac{1}{n} H_{\mu^{*}}\left(\left(\bigvee_{k=0}^{n-1} T^{-k} \alpha_{p} \vee \mathcal{C}\right)^{*} \mid \mathcal{C}^{*}\right) \\
& =\frac{1}{n} H_{\mu}\left(\bigvee_{k=0}^{n-1} T^{-k} \alpha_{p} \mid \mathcal{C}\right) \quad \text { by Lemma } 4.2 \\
& \leq \mu(A) h\left(A, \mathcal{B} \cap A, \mu_{A}, T_{A} \mid \mathcal{C} \cap A\right),
\end{aligned}
$$

by an earlier computation.

Putting things together, we can conclude that

$$
h\left(X^{*}, \mathcal{B}^{*}, \mu^{*}, T_{*} \mid \mathcal{C}^{*}\right) \leq \mu(A) h\left(A, \mathcal{B} \cap A, \mu_{A}, T_{A} \mid \mathcal{C} \cap A\right),
$$

which achieves the proof.

The following corollary is an immediate consequence of Proposition 7.1

Corollary 7.2. Let $(X, \mathcal{B}, \mu, T)$ be a $\mathbf{I I}_{\infty}$ system.

(1) If there exists some factor for which the Poisson and the Krengel entropy are equal, then the Poisson entropy of $(X, \mathcal{B}, \mu, T)$ is equal to its Krengel entropy.

(2) If there exists some extension for which the Poisson and the Krengel entropy are equal and finite, then the Poisson entropy of $(X, \mathcal{B}, \mu, T)$ is equal to its Krengel entropy.

(3) If there exists some factor for which the Poisson entropy is zero, then the Poisson entropy of $(X, \mathcal{B}, \mu, T)$ is equal to its Parry entropy.

(4) If there exists some factor for which the Krengel entropy is zero, then the Krengel entropy of $(X, \mathcal{B}, \mu, T)$ is equal to its Parry entropy.

Proof. The first two points are easy consequences of Proposition 7.1

To prove the third point, observe that if $\mathcal{C}$ is a factor on which the Poisson entropy is zero then, thanks to Proposition 7.1

$$
h\left(T^{*}\right)=\sup _{\mathcal{D} \subset \mathcal{B}, T_{*}^{-1} \mathcal{D}^{*} \subset \mathcal{D}^{*}} H_{\mu}\left(\mathcal{D}^{*} \mid T_{*}^{-1} \mathcal{D}^{*} \vee \mathcal{C}^{*}\right)
$$

which equals

$$
\sup _{\mathcal{D} \subset \mathcal{B}, T^{-1} \mathcal{D} \subset \mathcal{D}} H_{\mu}\left(\mathcal{D} \mid T^{-1} \mathcal{D} \vee \mathcal{C}\right)=\sup _{\mathcal{D} \subset \mathcal{B}, T^{-1} \mathcal{D} \subset \mathcal{D}} H_{\mu}\left(\mathcal{D} \vee \mathcal{C} \mid T^{-1}(\mathcal{D} \vee \mathcal{C})\right)
$$

by the same proposition. Therefore, $h\left(T^{*}\right) \leq h_{\mathrm{Pa}}(T)$, and the equality follows since Theorem 5.2 gives the other inequality.

The last point is proven with similar arguments. 
We point out that assertion (4) of Corollary 7.2, which concerns only Krengel and Parry entropy, is implied by [4].

\section{Additivity and SCALing of Poisson entropy}

We now show that just as with Krengel's entropy, the Poisson entropy of a sum of measures is the sum of the Poisson entropies, and scaling a measure by a positive constant scales the Poisson entropy.

Proposition 8.1. Suppose $\mu$ and $\nu$ are both T-invariant $\sigma$-finite measures on $(X, \mathcal{B})$, and $t, s>0$. We have

$$
h\left(X^{*}, \mathcal{B}^{*},(t \mu+s \nu)^{*}, T_{*}\right)=s \cdot h\left(X^{*}, \mathcal{B}^{*}, \mu^{*}, T_{*}\right)+t \cdot h\left(X^{*}, \mathcal{B}^{*}, \nu^{*}, T_{*}\right) .
$$

Proof. Let

$$
(\widehat{X}, \widehat{\mathcal{B}}, \lambda, \widehat{T}):=\left(X \times\{0,1\}, \mathcal{B} \times 2^{\{0,1\}}, \mu \times 1_{\{0\}}+\nu \times 1_{\{1\}}, T \times I d\right) .
$$

This system is isomorphic to the disjoint union of the two systems $(X, \mathcal{B}, \mu, T)$ and $(X, \mathcal{B}, \nu, T)$. The Poisson suspension of $(\widehat{X}, \widehat{\mathcal{B}}, \lambda, \widehat{T})$ is isomorphic to the product of the suspensions of $(X, \mathcal{B}, \mu, T)$ and $(X, \mathcal{B}, \nu, T)$. Thus,

$$
h\left(\widehat{X}^{*}, \widehat{\mathcal{B}}^{*}, \lambda^{*}, \widehat{T}_{*}\right)=h\left(X^{*}, \mathcal{B}^{*}, \mu^{*}, T_{*}\right)+h\left(X^{*}, \mathcal{B}^{*}, \nu^{*}, T_{*}\right) .
$$

Also, since $(X, \mathcal{B}, \mu+\nu, T)$ is a factor of $(\widehat{X}, \widehat{\mathcal{B}}, \lambda, \widehat{T})$, we have that the suspension of $(X, \mathcal{B}, \mu+\nu, T)$ is a factor of the suspension of $(\widehat{X}, \widehat{\mathcal{B}}, \lambda, \widehat{T})$. We thus see that

$$
h\left(X^{*}, \mathcal{B}^{*},(\mu+\nu)^{*}, T_{*}\right) \leq h\left(X^{*}, \mathcal{B}^{*}, \mu^{*}, T_{*}\right)+h\left(X^{*}, \mathcal{B}^{*}, \nu^{*}, T_{*}\right) .
$$

To prove that the above inequality is actually an equality, we observe that $(\widehat{X}, \widehat{\mathcal{B}}, \lambda$, $\widehat{T})$ is a bounded-to-one extension of $(X, \mathcal{B}, \mu+\nu, T)$ and is therefore a zero-entropy extension. By Proposition 7.1, it follows that $\left(\widehat{X}^{*}, \widehat{\mathcal{B}}^{*}, \lambda^{*}, \widehat{T}_{*}\right)$ is a zero-entropy extension of $\left(X^{*}, \mathcal{B}^{*},(\mu+\nu)^{*}, T_{*}\right)$.

We have just proved that Poisson entropy is additive, and it remains to prove that, for any $t>0$,

$$
h\left(X^{*}, \mathcal{B}^{*},(t \cdot \mu)^{*}, T_{*}\right)=t \cdot h\left(X^{*}, \mathcal{B}^{*}, \mu^{*}, T_{*}\right) .
$$

For rational $t$ 's, this follows from the above additivity property. If $t_{1}<t_{2}$, writing $t_{2} \cdot \mu=t_{1} \cdot \mu+\left(t_{2}-t_{1}\right) \cdot \mu$, we get $h\left(X^{*}, \mathcal{B}^{*},\left(t_{2} \cdot \mu\right)^{*}, T_{*}\right) \geq h\left(X^{*}, \mathcal{B}^{*},\left(t_{1} \cdot \mu\right)^{*}, T_{*}\right)$. So $t \rightarrow h\left(X^{*}, \mathcal{B}^{*},(t \cdot \mu)^{*}, T_{*}\right)$ is a monotone increasing function. Equation (6) now follows for any real $t>0$, since a monotone function which vanishes on the rational numbers is zero.

This result allows us to prove that the Poisson entropy of a squashable transformation is zero or infinite, just as Krengel and Parry entropy (recall that $(X, \mathcal{B}, \mu, T)$ is squashable if it is isomorphic to $(X, \mathcal{B}, c \mu, T)$ for a positive number $c \neq 1$ and completly squashable if this holds for any positive number $c$ ).

It has been conjectured that stochastic $\alpha$-semi-stable stationary processes have zero or infinite entropy. It is known in the case $\alpha=2$, which is the Gaussian case (see [5]), but remains unknown otherwise.

However, it has been noticed in [17] that $\alpha$-semi-stable stationary processes $(\alpha<$ 2) are factors of Poisson suspensions built over squashable systems (completely squashable in the stable case), associated with the Lévy measure of the process. Hence these Poisson suspensions are of zero or infinite entropy. Of course, this is not sufficient to prove the conjecture. 


\section{QUASI-FINITE CONSERVATIVE TRANSFORMATIONS}

9.1. Equality of the entropies. Recall the definition of a quasi-finite transformation from [11] (also see [2]): Let $(X, \mathcal{B}, \mu, T)$ be conservative measure-preserving. $A \in \mathcal{F}_{+}$is a quasi-finite set if $H_{\mu}\left(\rho_{A}\right)<\infty$, where $\rho_{A}$ is the first-return-time partition of $A$ :

$$
\rho_{A}:=\left\{A \cap\left(T^{-n} A \backslash \bigcup_{k=1}^{n-1} T^{-k} A\right), n \geq 1\right\} .
$$

$(X, \mathcal{B}, \mu, T)$ is quasi-finite if there exists a quasi-finite sweep-out set $A \in \mathcal{F}_{+}$. Using terminology similar to Aaronson and Park [2], we say that a local partition $\alpha$ is quasi-finite if it has a quasi-finite core $A$, and $\rho_{A} \prec \alpha$. We point out that conservative transformations which are not quasi-finite have been constructed in [2].

Parry has proved that, for quasi-finite transformations (called "pseudo-finite" in [13]), Krengel's definition of entropy coincides with Parry's. We show that, for such transformations, both are equal to the Poisson entropy.

Theorem 9.1. Let $(X, \mathcal{B}, \mu, T)$ be a quasi-finite measure-preserving transformation of type $\mathbf{I I}_{\infty}$. The Poisson entropy, the Krengel entropy and the Parry entropy of $(X, \mathcal{B}, \mu, T)$ are equal.

Proof. Let $\alpha$ be a local quasi-finite partition whose core $A$ is a sweep-out set. Applying Proposition 6.2, we get

$$
h\left(X^{*},(\hat{\alpha})^{*}, \mu^{*}, T_{*}\right) \leq \hat{h}(X, \mu, T, \alpha) .
$$

We want to show that $\hat{h}(X, \mu, T, \alpha)=H_{\mu}\left(\alpha_{0}^{\infty} \mid \alpha_{1}^{\infty}\right)$. The result follows by integrating (3) and by proving the convergence of $\int_{X} I_{\mu}\left(\alpha \mid \alpha_{1}^{n}\right) d \mu$ to $\int_{X} I_{\mu}\left(\alpha \mid \alpha_{1}^{\infty}\right) d \mu$ $=H_{\mu}\left(\alpha_{0}^{\infty} \mid \alpha_{1}^{\infty}\right)$. Remark that, since $\rho_{A} \prec \alpha, I_{\mu}\left(\alpha \mid \alpha_{1}^{n}\right)=I_{\mu}\left(\alpha \mid \alpha_{1}^{\infty}\right)=0$ on $X \backslash A$; therefore

$$
\int_{X} I_{\mu}\left(\alpha \mid \alpha_{1}^{n}\right) d \mu=\int_{A} I_{\mu}\left(\alpha \mid \alpha_{1}^{n}\right) d \mu .
$$

By Lemma 4.1 applied to the set $A$ with the restriction of the $\sigma$-algebras $\alpha$ and $\alpha_{1}^{n}$ to $A$, the right-hand side tends to $\int_{A} I_{\mu}\left(\alpha \mid \alpha_{1}^{\infty}\right) d \mu=\int_{X} I_{\mu}\left(\alpha \mid \alpha_{1}^{\infty}\right) d \mu$ which gives us the desired convergence.

Putting things together, we have proved

$$
h\left(X^{*},(\widehat{\alpha})^{*}, \mu^{*}, T_{*}\right) \leq H_{\mu}\left(\alpha_{0}^{\infty} \mid \alpha_{1}^{\infty}\right),
$$

the right-hand side being bounded by $h_{\mathrm{Pa}}(X, \widehat{\alpha}, \mu, T)$ by definition. By Theorem [5.2, the latter is in turn dominated by $h\left(X^{*},(\widehat{\alpha})^{*}, \mu^{*}, T_{*}\right)$. Hence, we obtain

$$
h\left(X^{*},(\widehat{\alpha})^{*}, \mu^{*}, T_{*}\right)=H_{\mu}\left(\alpha_{0}^{\infty} \mid \alpha_{1}^{\infty}\right)=h_{\mathrm{Pa}}(X, \widehat{\alpha}, \mu, T) .
$$

Now replace $\alpha$ by $\alpha_{n}$ in (8), where $\left(\alpha_{n}\right)$ is an increasing sequence of local quasifinite partitions with core $A$ having the property that $\left(\alpha_{n}\right)_{0}^{\infty} \uparrow \mathcal{B}$. Taking the limit in $n$, we obtain

$$
h\left(X^{*}, \mathcal{B}^{*}, \mu^{*}, T_{*}\right)=h_{\mathrm{Pa}}(X, \mathcal{B}, \mu, T),
$$

i.e. the Poisson entropy equals the Parry entropy. At last, the Krengel entropy equals the two others since the system is quasi-finite. 
9.2. Poisson suspensions of Markov chains. Poisson suspensions of Markov chains have been considered by several authors. Grabinsky [8 and Kalikow 9] have independently proved that the Poisson suspension of an ergodic, null-recurrent random walk is Bernoulli.

Let $\Sigma$ be a countable or finite set, and let $P=\left(p_{a, b}\right)_{a, b \in \Sigma}$ be a stochastic matrix which is irreducible and recurrent. As is well known, there exists a measure $q$ on $\Sigma$ which is stationary with respect to $P$, meaning $q P=q$, and this measure is unique up to scaling. The associated Markov shift is the system $(X, \mathcal{B}, \mu, T)$, where $X=\Sigma^{\mathbb{Z}}, T: X \rightarrow X$ denotes the shift map $\left(T(x)_{n}=x_{n+1}\right), \mathcal{B}$ denotes the Borel $\sigma$-algebra of $X$ with respect to the product topology and $\mu$ is given by

$$
\mu\left(\left[a_{1}, \ldots, a_{k}\right]\right)=q_{a_{1}} \prod_{i=2}^{k} p_{a_{i-1}, a_{i}} .
$$

Based on the Krengel entropy of recurrent Markov chains and our previous result about Poisson entropy of quasi-finite transformations, we have

Corollary 9.2. The entropy of the Poisson suspension of a recurrent Markov shift with transition matrix $P=\left(p_{a, b}\right)_{a, b \in \Sigma}$ and stationary measure $q$ is given by

$$
h\left(X^{*}, \mathcal{B}^{*}, \mu^{*}, T^{*}\right)=\sum_{a \in \Sigma} q_{a} \sum_{b \in \Sigma} p_{a, b} \log \frac{1}{p_{a, b}} .
$$

Proof. By Krengel's formula (Theorem 4.1 of [11]), the Krengel entropy of $(X, \mathcal{B}, \mu$, $T$ ) is given by the right-hand side of (9). By taking the standard Markov partition $\xi$, we see that

$$
H\left(\xi_{-\infty}^{0} \mid T^{-1} \xi_{-\infty}^{0}\right)=\sum_{a \in \Sigma} q_{a} \sum_{b \in \Sigma} p_{a, b} \log \frac{1}{p_{a, b}} .
$$

Thus, Parry's entropy dominates Krengel's. Hence both are equal.

Without loss of generality we can assume that the transition matrix is irreducible. In the particular case when $(X, \mathcal{B}, \mu, T)$ is a renewal system $\left(\Sigma=\mathbb{N}\right.$ and $p_{n, n-1}=$ 1 for all $n>1$ ), the right-hand side of (9) is simply the entropy of the firstreturn-time partition of the state 1 . Hence, if the Krengel entropy is finite, the renewal system is quasi-finite, in which case the Poisson entropy is equal to the Krengel entropy by Theorem 9.1. Otherwise, the Parry entropy is infinite, and by Theorem 5.2 it is equal to the Poisson entropy. Now it remains to note that every irreducible recurrent Markov chain has a factor which is a renewal system, and apply Corollary 7.2 (1).

In [8, it is claimed that the entropy of the Poisson suspension of a null-recurrent Markov chain is infinite (Proposition 4.3). Corollary 9.2, together with the existence of such chains with finite Krengel entropy (see [11]), contradict this result. The mistake in 8 comes from the following incorrect assertion which Grabinsky invokes in the proof of Proposition 4.3, to bound from below the entropy of a certain partition: If $\xi$ is a Markov partition (i.e. $\xi$ is independent of $\xi_{-\infty}^{-1}$ given $T^{-1} \xi$ ) and $\eta$ a partition which is measurable with respect to $\xi$, then $\eta^{*}$ is a Markov partition as well. It would imply, in particular, that given the number of particles in a certain Markov state $A$ at time -1 , the number of particles in $A$ at time 0 is independent of the number of particles in $A$ at time -2 . 


\section{Zero Poisson Entropy}

In this section, we prove that some class of cutting-and-stacking constructions (including finite-rank transformations) and transformations without a countable Lebesgue component in their spectrum both have zero Poisson entropy. It is well known that rank one transformations also have zero Krengel entropy, therefore, Krengel entropy, Parry entropy and Poisson entropy are equal in this case.

The construction of a non-quasi-finite transformation in [2] is of this kind, so the results of this section do not follow from Theorem 9.1 .

10.1. Cutting-and-stacking constructions. A cutting-and-stacking construction is an iterative method to present conservative transformations. We briefly describe this construction and refer to Friedman's book [6] for details.

A column of height $h \in \mathbb{N}$ is an array $I_{1}, \ldots, I_{h}$ of pairwise disjoint intervals of the same length, considered as "stacked" one on top of the other. At stage $n$ of the cutting-and-stacking procedure, the $n$-th tower $X_{n}$ consists of $c_{n}$ columns of heights $\left\{h_{n, i}\right\}_{1 \leq i \leq c_{n}}$ and equal width. The transformation acts by translating each interval to the interval above it. At stage $n$, the transformation is undefined for points on the top intervals. At stage $n+1$, each column is "cut" into $k_{n}$ columns, all the columns are "stacked" one on top of the other, and then the newly formed column is cut into $c_{n+1}$ columns. Then some new intervals are possibly added on the top of every column. As the length of the intervals at stage $n$ tends to 0 , the measure of the points on which the transformation is undefined at stage $n$ tends to 0 . Such a construction is said to have rank one if $c_{n}=1$ for every $n \geq 1$, and finite rank if $\left\{c_{n}\right\}$ is bounded.

Denote by $\epsilon_{n}$ the length of the intervals at stage $n$. Clearly, $\epsilon_{n} \rightarrow 0$ as $n \rightarrow \infty$.

Proposition 10.1. Let $(X, \mathcal{B}, \mu, T)$ be a cutting-and-stacking construction as above. If $c_{n} \epsilon_{n} \log \epsilon_{n} \rightarrow 0$ as $n \rightarrow \infty$, then $h\left(X^{*}, \mathcal{B}^{*}, \mu^{*}, T_{*}\right)=0$. In particular, this is the case if $T$ has finite rank.

Proof. Let $\beta_{n}=\left\{I_{n, 1}, \ldots, I_{n, c_{n}}\right\}$ denote the set of intervals composing the base of the tower at stage $n$, and let

$$
\xi_{n}=\left\{I_{n, 1}, T I_{n, 1}, \ldots, T^{h_{n, 1}-1} I_{n, 1}, \ldots, I_{n, c_{n}}, \ldots, T^{h_{n, c_{n}}-1} I_{n, c_{n}}\right\}
$$

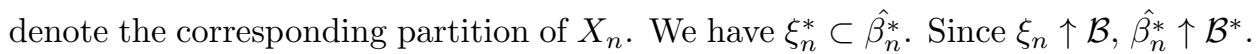
From this, we deduce that $h_{\mu^{*}}\left(T_{*}, \hat{\beta_{n}^{*}}\right) \uparrow h\left(X^{*}, \mathcal{B}^{*}, \mu^{*}, T_{*}\right)$. But $h_{\mu^{*}}\left(T_{*}, \hat{\beta_{n}^{*}}\right) \leq$ $H_{\mu^{*}}\left(\beta_{n}^{*}\right)=c_{n} f\left(\epsilon_{n}\right) \sim-c_{n} \epsilon_{n} \log \epsilon_{n}$, where $f(x)$ denotes the entropy function of a Poisson variable with parameter $x$.

10.2. Spectral criterion. The following proposition and corollary give a spectral criterion for positive Poisson entropy. The corresponding result about Kolmogorov entropy is well known in the finite measure case.

Proposition 10.2. If $(X, \mathcal{B}, \mu, T)$ is of type $\mathbf{I I}_{\infty}$ and has positive Poisson entropy, then its spectrum has a component which is countable Lebesgue.

Proof. Pick a sweep out set $A \in \mathcal{B}$ with small measure in order to have $H_{\mu^{*}}\left(\xi_{0}^{*}\right)=$ $f(\mu(A))<h\left(T_{*}\right)$, where $\xi_{0}$ is the partition $\left\{A, A^{c}\right\}$ of $X$. We now refine the local partition $\xi_{0}$ by increasing finite local partitions $\xi_{n}$ so that $\xi_{n} \uparrow \mathcal{B}$, which implies $h\left(T_{*}, \xi_{n}^{*}\right) \uparrow h\left(T_{*}\right)$. Using the continuity of Kolmogorov entropy, the continuity of 
$f$ and the continuity of $\mu$, we can assume that this increasing sequence $\xi_{n}$ is such that

$$
0<h\left(T_{*}, \xi_{1}^{*}\right)<\cdots<h\left(T_{*}, \xi_{n}^{*}\right)<\cdots .
$$

In the following, for a $\sigma$-algebra $\mathcal{A} \subset \mathcal{B}^{*}$, we denote by $L^{2}(\mathcal{A})$ the corresponding linear subspace of $L^{2}\left(\mu^{*}\right)$ of square-integrable $\mathcal{A}$-measurable functions, and $U_{T_{*}}$ is the unitary operator induced from $T_{*}$. Denote by $\mathfrak{C}$ the first chaos of $L^{2}\left(\mu^{*}\right)$, i.e. the closure of the linear subspace generated by $N(A)-\mu(A), A \in \mathcal{B}, \mu(A)<\infty$. The arguments below are classical. We already know that the suspension has a countable Lebesgue component in its spectrum; however, to get the result, we will see that a countable Lebesgue component is localized in $\mathfrak{C}$ which is unitary isomorphic to $L^{2}(\mu)$. Set $H_{1}:=L^{2}\left(\left(\xi_{1}^{*}\right)_{-\infty}^{0}\right) \cap \mathfrak{C}$ and note that it is non-empty since it contains the functions $N(A)-\mu(A), A \in T^{-k} \xi_{1}, k \in \mathbb{N}$. Remark that $U_{T_{*}^{-1}} H_{1} \subset H_{1}$ and that we cannot have $U_{T_{*}^{-1}} H_{1}=H_{1}$ since it would imply that $\sigma\left(H_{1}\right)$ belongs to the Pinsker factor of $T_{*}$, and, as the factor $\widehat{\xi_{1}^{*}}$ is generated (as a $\sigma$ algebra) by $\bigcup_{n>0} U_{T_{*}^{n}} H_{1}=H_{1}, h\left(T_{*}, \xi_{1}^{*}\right)=0$, which is a contradiction. Functions belonging to $V_{1}:=H_{1} \ominus U_{T_{*}^{-1}} H_{1}$ have Lebesgue spectral measure.

Set $H_{2}:=\left(L^{2}\left(\left(\xi_{2}^{*}\right)_{-\infty}^{0}\right) \cap \mathfrak{C}\right) \cap\left(L^{2}\left(\widehat{\xi_{1}^{*}}\right) \cap \mathfrak{C}\right)^{\perp}$. It is also non-empty since $\bigcup_{n \geq 0} U_{T_{*}^{n}}\left(L^{2}\left(\left(\xi_{2}^{*}\right)_{-\infty}^{0}\right) \cap \mathfrak{C}\right)$ generates $\widehat{\xi_{2}^{*}}$, which is strictly larger than $\widehat{\xi_{1}^{*}}$ because $h\left(T_{*}, \xi_{1}^{*}\right)<h\left(T_{*}, \xi_{2}^{*}\right)$. Moreover, we have $U_{T^{-1}} H_{2} \subset H_{2}$, and once again, we cannot have $U_{T_{*}^{-1}} H_{2}=H_{2}$, as it would imply that $\sigma\left(H_{2}\right)$ belongs to the Pinsker factor of $T_{*}$, and this is impossible since the entropy of $\widehat{\xi_{2}^{*}}=\widehat{\xi_{1}^{*}} \vee \sigma\left(H_{2}\right)$ would be equal to that of $\widehat{\xi_{1}^{*}}$, which is a contradiction. Therefore we can set $V_{2}:=H_{2} \ominus U_{T_{*}^{-1}} H_{2}$, which is constituted by functions having Lebesgue spectral measure and satisfies $\overline{\bigcup_{n \in \mathbb{Z}} U_{T_{*}^{n}} V_{2}} \perp \overline{\bigcup_{n \in \mathbb{Z}} U_{T_{*}^{n}} V_{1}}$.

Proceeding by induction, we construct an infinite sequence of mutually orthogonal invariant subspaces $\overline{\bigcup_{n \in \mathbb{Z}} U_{T_{*}^{n}} V_{n}}$ of $\mathfrak{C}$ on which $U_{T_{*}}$ has Lebesgue maximal spectral type. Thanks to the unitary isomorphism between $\mathfrak{C}$ and $L^{2}(\mu)$, these subspaces can be transferred into $L^{2}(\mu)$, and we have proved that $T$ has a countable Lebesgue component in its spectrum.

Corollary 10.3. If the maximal spectral type of $T$ is singular or if $T$ has finite multiplicity, then its Poisson entropy is zero.

We mention that Parry 12 has shown that a $K$-automorphism has countable Lebesgue spectrum. From his proof one can easily obtain that if the maximal spectral type is singular or has finite multiplicity, then the Parry entropy is zero. Since zero Poisson entropy implies zero Parry entropy, our above corollary allows us to recover Parry's result. We do not know of a sufficient spectral criterion for zero Krengel entropy.

\section{Perfect Poissonian partitions and the Poisson-Pinsker factor}

For a probability-preserving system $(X, \mathcal{B}, \mu, T)$, the Pinsker factor, denoted by $\mathcal{P}(T)$, is the maximum factor ( $T$-sub-invariant $\sigma$-algebra) with zero entropy. For each of the various notions of entropy for $\sigma$-finite transformations, we generalize this definition: We say that a factor is Pinsker if it is the maximum zero entropy factor. We can thus speak of a Krengel-Pinsker factor, a Parry-Pinsker factor and 
a Poisson-Pinsker factor of a conservative transformation. The existence of these is not obvious in general. The following proposition gives a sufficient condition for Pinsker factors to exist, which we later show to be necessary as well:

Proposition 11.1. Let $(X, \mathcal{B}, \mu, T)$ be an ergodic type $\mathbf{I I}_{\infty}$ system. Assume the Pinsker factor $\mathcal{P}\left(T_{*}\right)$ of the Poisson suspension is of the form $\mathcal{P}^{*}$ for some $\sigma$-finite $\sigma$-algebra $\mathcal{P}$. Then $\mathcal{P}$ is both the Poisson-Pinsker and the Parry-Pinsker factor of T. Moreover, if there exists a factor with zero Krengel entropy, then $\mathcal{P}$ is also the Krengel-Pinsker factor.

Proof. Since $\mathcal{P}^{*}$ is the Pinsker factor of $T_{*}$, for any factor $\mathcal{C}$ of zero Poisson entropy, $\mathcal{C}^{*} \subset \mathcal{P}^{*}$. But the conditional expectations $\pi_{\mathcal{C}^{*}}$ and $\pi_{\mathcal{P}^{*}}$ of $\mathcal{C}^{*}$ and $\mathcal{P}^{*}$ are the exponential of the conditional expectations $\pi_{\mathcal{C}}$ and $\pi_{\mathcal{P}}$ of $\mathcal{C}$ and $\mathcal{P}$ (see [18); therefore as $\pi_{\mathcal{C}^{*}} \leq \pi_{\mathcal{P}^{*}}$, we get $\pi_{\mathcal{C}} \leq \pi_{\mathcal{P}}$, which means that $\mathcal{C} \subset \mathcal{P}$, as $\mathcal{C}$ is $\sigma$-finite. This shows that $\mathcal{P}$ is the Poisson-Pinsker factor of $(X, \mathcal{B}, \mu, T)$.

We now prove that $\mathcal{P}$ is also the Parry-Pinsker factor. Assume that $\mathcal{C}$ is a factor of zero Parry entropy. By Lemma 2.2 we have $\mathcal{P}^{*} \cap \mathcal{C}^{*}=(\mathcal{P} \cap \mathcal{C})^{*}$. Moreover, if $\mathcal{P} \cap \mathcal{C}$ contains no sets of positive finite measure, then $(\mathcal{P} \cap \mathcal{C})^{*}$ is trivial, and since $\mathcal{P}^{*}$ is the Pinsker factor of $\left(X^{*}, \mathcal{B}^{*}, \mu^{*}, T_{*}\right)$, then $\mathcal{C}^{*}$ is a $K$-system. By a well-known disjointness result (for probability-preserving transformations), $\mathcal{P}^{*}$ and $\mathcal{C}^{*}$ are independent. For any two positive and finite measure sets $A \in \mathcal{P}$ and $B \in \mathcal{C}$, we have

$$
\int_{X^{*}}(\gamma(A)-\mu(A))(\gamma(B)-\mu(B)) \mu^{*}(d \gamma)=0 .
$$

But the left-hand side equals $\mu(A \cap B)$, so $A$ and $B$ are disjoint $\bmod \mu$. This is impossible for all $A \in \mathcal{P}$ and $B \in \mathcal{C}$ because it would contradict the fact that $\mathcal{P}$ is $\sigma$-finite and $T$ is of type $\mathbf{I I}_{\infty}$. Indeed the measurable union of the sets of finite positive measure contained in $\mathcal{C}$ would be an infinite measure atom of $\mathcal{P}$, which is impossible by assumption. This shows that $\mathcal{P} \cap \mathcal{C}$ must contain a set of positive, finite measure, so by ergodicity it is $\sigma$-finite. Thus, $\mathcal{P} \cap \mathcal{C}$ is a factor of $\mathcal{C}$ with zero Poisson entropy. Thanks to Corollary 7.2 (3), Parry and Poisson entropy coincide on $\mathcal{C}$, and since the first one is zero, the second one is zero. This implies that $\mathcal{C} \subset \mathcal{P}$. Hence $\mathcal{P}$ is also the Parry-Pinsker factor. The statement about the Krengel-Pinsker factor is proved in the same way, using Corollary 7.2 (4).

Recall that a $\sigma$-algebra $\xi$ for an invertible probability-preserving system $(\Omega, \mathcal{F}, m$, $T)$ is called a perfect $\sigma$-algebra if $T^{-1} \xi \subset \xi, \widehat{\xi}=\mathcal{B}, h(T)=H_{m}\left(\xi \mid T^{-1} \xi\right)$ and

$$
\bigcap_{n=0}^{\infty} T^{-n} \xi=\mathcal{P}(\Omega, \mathcal{F}, m, T) .
$$

In fact, if the entropy is finite, the last condition is a consequence of the others as proved in [15] (p. 38, claim 12.4):

Lemma 11.2. Assume $(\Omega, \mathcal{F}, m, T)$ is an invertible probability-preserving system. If $T^{-1} \xi \subset \xi, \widehat{\xi}=\mathcal{B}$ and $h(T)=H_{m}\left(\xi \mid T^{-1} \xi\right)<\infty$, then

$$
\bigcap_{n=0}^{\infty} T^{-n} \xi=\mathcal{P}(\Omega, \mathcal{F}, m, T) .
$$

Let us introduce the following definition: A $\sigma$-finite $\sigma$-algebra $\mathcal{A}$ is said to be entropy determining (ED) if $\mathcal{A}^{*}$ is perfect with respect to the factor it generates. 
Observe that on the factor generated by an ED $\sigma$-algebra $\mathcal{A}$, Parry and Poisson entropies coincide:

$$
h_{\mathrm{Pa}}(T, \widehat{\mathcal{A}}) \leq h\left(T_{*}, \mathcal{A}^{*}\right)=H_{\mu^{*}}\left(\mathcal{A}^{*} \mid T_{*}^{-1} \mathcal{A}^{*}\right)=H_{\mu}\left(\mathcal{A} \mid T^{-1} \mathcal{A}\right) \leq h_{\mathrm{Pa}}(T, \widehat{\mathcal{A}}) .
$$

The class of ED $\sigma$-algebras plays the same role as finite-entropy partitions do in the finite-measure case.

We are now ready to prove a "Poisson analogue" of the Rokhlin-Sinaï Theorem regarding Pinsker factors of probability-preserving transformations. We recall that $T$ is remotely infinite if there exists a $\sigma$-finite sub- $\sigma$-algebra $\alpha$ such that $T^{-1} \alpha \subset \alpha$, $T^{n} \alpha \uparrow \mathcal{B}$ and $T^{-n} \alpha \downarrow \mathcal{G}(\bmod \mu)$, where $\mathcal{G}$ has no set of positive finite measure. Also recall that a probability-preserving transformation is a $K$-system if and only if there exists a sub-invariant generating $\sigma$-algebra with a trivial tail. The notion of a remotely-infinite system can be considered as an infinite-measure analogue of a probability-preserving $K$-system. The Rokhlin-Sinaï Theorem tells us that a probability-preserving transformation is a $K$-system if and only if the trivial factor is the only factor of zero entropy.

Theorem 11.3. Let $(X, \mathcal{B}, \mu, T)$ be an ergodic system of type $\mathbf{I I}_{\infty}$. Assume there exists an ED partition $\mathcal{A}$ such that $H_{\mu}\left(\mathcal{A} \mid T^{-1} \mathcal{A}\right)<\infty$. Then

- there exists a generating ED partition (in particular $h_{P a}(T)=h\left(T_{*}\right)$ ).

- $T$ is either remotely infinite or $\mathcal{P}\left(T_{*}\right)$ is Poissonian: $\mathcal{P}\left(T_{*}\right)=\mathcal{P}^{*}$ for some $\sigma$-finite $T$-invariant $\sigma$-algebra $\mathcal{P}$. In the latter case, $\mathcal{P}$ is the Poisson (and Parry) Pinsker factor of $T$.

Proof. Let $\xi$ be a finite local partition with a sweep-out core $A \in \mathcal{A}$. We first show that $\xi_{-\infty}^{0} \vee \mathcal{A}$ is also an ED $\sigma$-algebra. On the one hand, we have

$$
\begin{aligned}
& H_{\mu^{*}}\left(\left(\left(\xi_{-p}^{0}\right)^{*} \vee \mathcal{A}^{*}\right)_{0}^{n} \mid \mathcal{A}^{*}\right) \\
& =H_{\mu^{*}}\left(\left(\left(\xi_{-p}^{0}\right)^{*}\right)_{0}^{n} \mid\left(\mathcal{A}^{*}\right)_{0}^{n}\right)+H_{\mu^{*}}\left(\left(\mathcal{A}^{*}\right)_{0}^{n} \mid \mathcal{A}^{*}\right) \\
& =H_{\mu^{*}}\left(\left(\left(\xi_{-p}^{0}\right)^{*}\right) \mid \mathcal{A}^{*}\right)+\sum_{k=1}^{n} H_{\mu^{*}}\left(\left(\xi_{-p}^{0}\right)^{*} \vee \mathcal{A}^{*} \mid T_{*}^{-1}\left(\left(\xi_{-p}^{0}\right)^{*}\right)_{-k}^{0} \vee T_{*}^{k} \mathcal{A}^{*}\right) \\
& +n H_{\mu^{*}}\left(\mathcal{A}^{*} \mid T_{*}^{-1} \mathcal{A}^{*}\right) .
\end{aligned}
$$

Dividing by $n$, this tends to

$$
H_{\mu^{*}}\left(\left(\xi_{-p}^{0}\right)^{*} \mid T_{*}^{-1}\left(\left(\xi_{-p}^{0}\right)^{*}\right)_{-\infty}^{0} \vee \widehat{\mathcal{A}^{*}}\right)+H_{\mu^{*}}\left(\mathcal{A}^{*} \mid T_{*}^{-1} \mathcal{A}^{*}\right)
$$

Since $\mathcal{A}$ is $\mathrm{ED}$, the second term equals $h\left(T_{*}, \mathcal{A}^{*}\right)$. Thus the previous expression equals $h\left(T_{*},\left(\xi_{-p}^{0}\right)^{*} \vee \mathcal{A}^{*}\right)$. 
On the other hand,

$$
\begin{aligned}
& H_{\mu}\left(\left(\xi_{-p}^{0} \vee \mathcal{A}\right)_{0}^{n} \mid \mathcal{A}\right) \\
& =H_{\mu}\left(\xi_{-p}^{0} \vee \mathcal{A} \mid \mathcal{A}\right)+\sum_{k=1}^{n} H_{\mu}\left(T^{k}\left(\xi_{-p}^{0} \vee \mathcal{A}\right) \mid\left(\bigvee_{j=0}^{k-1} T^{j}\left(\xi_{-p}^{0} \vee \mathcal{A}\right)\right) \vee \mathcal{A}\right) \\
& =H_{\mu}\left(\xi_{-p}^{0} \vee \mathcal{A} \mid \mathcal{A}\right)+\sum_{k=1}^{n} H_{\mu}\left(T^{k}\left(\xi_{-p}^{0} \vee \mathcal{A}\right) \mid\left(\bigvee_{j=0}^{k-1} T^{j} \xi_{-p}^{0}\right) \vee T^{k-1} \mathcal{A}\right) \\
& =H_{\mu}\left(\xi_{-p}^{0} \vee \mathcal{A} \mid \mathcal{A}\right)+\sum_{k=1}^{n} H_{\mu}\left(T^{k}\left(\xi_{-p}^{0} \vee \mathcal{A}\right) \mid T^{k}\left(\left(\bigvee_{j=-k}^{-1} T^{j} \xi_{-p}^{0}\right) \vee T^{-1} \mathcal{A}\right)\right) \\
& =H_{\mu}\left(\xi_{-p}^{0} \vee \mathcal{A} \mid \mathcal{A}\right)+\sum_{k=1}^{n} H_{\mu}\left(\xi_{-p}^{0} \vee \mathcal{A} \mid T^{-1}\left(\xi_{-p}^{0}\right)_{-k}^{0} \vee T^{-1} \mathcal{A}\right)
\end{aligned}
$$

which, divided by $n$, tends to $H_{\mu}\left(\xi_{-p}^{0} \vee \mathcal{A} \mid T^{-1}\left(\xi_{-\infty}^{0} \vee \mathcal{A}\right)\right)$.

But observe that

$$
\begin{aligned}
H_{\mu^{*}}\left(\left(\left(\xi_{-p}^{0}\right)^{*} \vee \mathcal{A}^{*}\right)_{0}^{n} \mid \mathcal{A}^{*}\right) & \leq H_{\mu^{*}}\left(\left(\left(\xi_{-p}^{0} \vee \mathcal{A}\right)^{*}\right)_{0}^{n} \mid \mathcal{A}^{*}\right) \\
\leq H_{\mu^{*}}\left(\left(\left(\xi_{-p}^{0} \vee \mathcal{A}\right)_{0}^{n}\right)^{*} \mid \mathcal{A}^{*}\right) & =H_{\mu}\left(\left(\xi_{-p}^{0} \vee \mathcal{A}\right)_{0}^{n} \mid \mathcal{A}\right) .
\end{aligned}
$$

Therefore, we have for all $p$

$$
\begin{aligned}
& h\left(T_{*},\left(\xi_{-p}^{0}\right)^{*} \vee \mathcal{A}^{*}\right) \leq H_{\mu}\left(\xi_{-p}^{0} \vee \mathcal{A} \mid T^{-1}\left(\xi_{-\infty}^{0} \vee \mathcal{A}\right)\right) \\
& \quad=H_{\mu^{*}}\left(\left(\xi_{-p}^{0} \vee \mathcal{A}\right)^{*} \mid T_{*}^{-1}\left(\xi_{-\infty}^{0} \vee \mathcal{A}\right)^{*}\right)
\end{aligned}
$$

and we deduce that

$$
h\left(T_{*},\left(\xi_{-\infty}^{0}\right)^{*} \vee \mathcal{A}^{*}\right) \leq H_{\mu^{*}}\left(\left(\xi_{-\infty}^{0} \vee \mathcal{A}\right)^{*} \mid T_{*}^{-1}\left(\xi_{-\infty}^{0} \vee \mathcal{A}\right)^{*}\right) .
$$

But $\xi_{-\infty}^{0} \cap \mathcal{A}$ is non-empty (since it contains $A$ ), and as $T$ is $\mathbf{I I}_{\infty}$, it is also nonatomic. Therefore, $\left(\xi_{-\infty}^{0}\right)^{*} \vee \mathcal{A}^{*}=\left(\xi_{-\infty}^{0} \vee \mathcal{A}\right)^{*}$ by Lemma 2.3, and

$$
h\left(T_{*},\left(\xi_{-\infty}^{0} \vee \mathcal{A}\right)^{*}\right) \leq H_{\mu^{*}}\left(\left(\xi_{-\infty}^{0} \vee \mathcal{A}\right)^{*} \mid T_{*}^{-1}\left(\xi_{-\infty}^{0} \vee \mathcal{A}\right)^{*}\right) .
$$

Since we have the other inequality, we can conclude that

$$
h\left(T_{*},\left(\xi_{-\infty}^{0} \vee \mathcal{A}\right)^{*}\right)=H_{\mu^{*}}\left(\left(\xi_{-\infty}^{0} \vee \mathcal{A}\right)^{*} \mid T_{*}^{-1}\left(\xi_{-\infty}^{0} \vee \mathcal{A}\right)^{*}\right)<\infty ;
$$

thus $\xi_{-\infty}^{0} \vee \mathcal{A}$ is ED.

Using this preliminary result, by considering an increasing sequence $\left(\xi_{k}\right)$ of finite local partitions with core $A$ such that $\widehat{\xi_{k}} \uparrow \mathcal{B}$, we build an increasing sequence of ED partitions $\left(\left(\xi_{k}\right)_{-\infty}^{0} \vee \mathcal{A}\right)$.

By definition, each $\left(\left(\xi_{k}\right)_{-\infty}^{0} \vee \mathcal{A}\right)^{*}$ is a perfect $\sigma$-algebra for the corresponding factor. In particular, $\bigcap_{n} T_{*}^{-n}\left(\left(\xi_{k}\right)_{-\infty}^{0} \vee \mathcal{A}\right)^{*}$ is a zero-entropy factor of $T_{*}$. As 
$h\left(T_{*},\left(\left(\xi_{k}\right)_{-\infty}^{0} \vee \mathcal{A}\right)^{*}\right)$ is finite for all $k$, we can inductively define a sequence $\eta_{k}=\eta_{k-1} \vee T_{*}^{-n_{k}}\left(\left(\xi_{k}\right)_{-\infty}^{0} \vee \mathcal{A}\right)^{*}$, where the integers $\left\{n_{k}\right\}$ are chosen so that

$$
H_{\mu^{*}}\left(\eta_{i} \mid\left(\eta_{j-1}\right)_{-\infty}^{-1}\right)-H_{\mu^{*}}\left(\eta_{i} \mid\left(\eta_{j}\right)_{-\infty}^{-1}\right)<\frac{1}{i} 2^{j-i}
$$

whenever $i<j$. Proceeding as in page 69 of [14], we obtain that $\eta:=\bigvee_{k \geq 1} \eta_{k}$ is a perfect $\sigma$-algebra for $T_{*}$. We have to show that $\eta$ is indeed a Poissonian $\sigma$-algebra. Observe that $T_{*}^{-n_{k}}\left(\left(\xi_{k}\right)_{-\infty}^{0} \vee \mathcal{A}\right)^{*}=\left(T^{-n_{k}}\left(\left(\xi_{k}\right)_{-\infty}^{0} \vee \mathcal{A}\right)\right)^{*}$ and thus

$$
\eta_{k}=\bigvee_{j=0}^{k}\left(T^{-n_{j}}\left(\left(\xi_{j}\right)_{-\infty}^{0} \vee \mathcal{A}\right)\right)^{*} .
$$

For any $k \geq 0, T^{-n_{k}}\left(\left(\xi_{0}\right)_{-\infty}^{0} \vee \mathcal{A}\right)$ is non-atomic, and for any $j \leq k$,

$$
T^{-n_{k}}\left(\left(\xi_{0}\right)_{-\infty}^{0} \vee \mathcal{A}\right) \subset T^{-n_{j}}\left(\left(\xi_{j}\right)_{-\infty}^{0} \vee \mathcal{A}\right) \text {. }
$$

We can apply Lemma 2.3 to get

$$
\eta_{k}=\left(\bigvee_{j=0}^{k} T^{-n_{j}}\left(\left(\xi_{j}\right)_{-\infty}^{0} \vee \mathcal{A}\right)\right)^{*} .
$$

Setting $\alpha_{k}=\bigvee_{j=0}^{k} T^{-n_{j}}\left(\left(\xi_{j}\right)_{-\infty}^{0} \vee \mathcal{A}\right)$ and $\alpha=\bigvee_{k \geq 1} \alpha_{k}$, then $\alpha_{k} \uparrow \alpha$ and so $\eta_{k}=\alpha_{k}^{*} \uparrow \alpha^{*}$. We conclude that $\eta=\alpha^{*}$, and so $\alpha$ is a generating ED partition.

Using the fact that $\eta$ is a generating perfect $\sigma$-algebra, and applying Lemma 2.4. we obtain

$$
\mathcal{P}\left(T_{*}\right)=\bigcap_{n=0}^{\infty} T_{*}^{-n} \eta=\left(\bigcap_{n=0}^{\infty} T^{-n} \alpha\right)^{*} .
$$

In the case where $\bigcap_{n=0}^{\infty} T^{-n} \alpha$ is $\sigma$-finite, $\mathcal{P}\left(T_{*}\right)$ is indeed Poissonian. Otherwise, by ergodicity of $T, \bigcap_{n=0}^{\infty} T^{-n} \alpha$ contains no set of positive finite measure and $T$ is remotely infinite.

We remark that whenever $(X, \mathcal{B}, \mu, T)$ is of type $\mathbf{I I}_{\infty}$, but not necessarily ergodic, by the ergodic decomposition we can uniquely decompose $\mu=\mu_{0}+\mu_{1}$, where $\mu_{1}$ and $\mu_{2}$ are mutually singular and both are $T$-invariant, $\left(X, \mathcal{B}, \mu_{0}, T\right)$ is remotely infinite and $\left(X, \mathcal{B}, \mu_{1}, T\right)$ has a Poisson-Pinsker factor as above.

Concluding this section, we state the following proposition, which along with Proposition 11.1 and Theorem 11.3 completes the picture about Poisson-Pinkser factors:

Proposition 11.4. Let $(X, \mathcal{B}, \mu, T)$ be an ergodic $\mathbf{I I}_{\infty}$-system with a zero Poisson entropy factor $\mathcal{A}$. Then there exists a generating ED partition. In particular, $(X, \mathcal{B}, \mu, T)$ possesses a Poisson-Pinsker factor $\mathcal{P}$, and $\mathcal{P}^{*}$ is the Pinsker factor of $T_{*}$.

Proof. $\mathcal{A}$ is an ED partition which satisfies $H\left(\mathcal{A} \mid T^{-1} \mathcal{A}\right)=0$; therefore, this is a direct application of Theorem 11.3 . 


\section{Some more Results, REMARKS AND QUESTIONS}

The conclusion of Proposition 11.4 above yields a natural question: Does the non-triviality of the Pinsker factor of $T_{*}$ imply the existence of a Poisson-Pinsker factor for $T$ ? We can only partially answer this question:

Proposition 12.1. Assume $T$ is an ergodic $\mathbf{I I}_{\infty}$-system which satisfies $h\left(T_{*}\right)=$ $h_{P a}(T)<\infty$. If $\mathcal{P}\left(T_{*}\right) \neq\left\{X^{*}, \emptyset\right\}$, then $T$ possesses a Poisson-Pinsker factor $\mathcal{P}$ and $\mathcal{P}^{*}=\mathcal{P}\left(T_{*}\right)$.

Proof. First we are going to show that

$$
h_{\mathrm{Pa}}(T)=\sup \left\{H\left(\mathcal{A} \mid T^{-1} \mathcal{A}\right), \mathcal{A} \subset T^{-1} \mathcal{A}, \widehat{\mathcal{A}}=\mathcal{B}\right\} .
$$

It is based on the following observation: Consider an increasing $\sigma$-algebra $\mathcal{A}$, such that $\widehat{\mathcal{A}} \neq \mathcal{B}$, a set $A \in \mathcal{A}$ and a finite local partition $\xi$ such that $\widehat{\mathcal{A} \vee \xi}=\mathcal{B}$ (the existence of such a partition is ensured by Theorem 2.5 in [4], as the extension $\mathcal{B} \rightarrow \mathcal{A}$ has finite relative Poisson entropy and therefore finite relative Krengel entropy by Proposition 7.1). From earlier computations, we have

$$
\begin{gathered}
\frac{1}{n} H_{\mu^{*}}\left(\left(\left(\xi_{-p}^{0}\right)^{*} \vee \mathcal{A}^{*}\right)_{0}^{n} \mid \mathcal{A}^{*}\right) \\
\rightarrow H_{\mu^{*}}\left(\left(\xi_{-p}^{0}\right)^{*} \mid T_{*}^{-1}\left(\left(\xi_{-p}^{0}\right)^{*}\right)_{-\infty}^{0} \vee \widehat{\mathcal{A}^{*}}\right)+H_{\mu}\left(\mathcal{A} \mid T^{-1} \mathcal{A}\right)
\end{gathered}
$$

and

$$
\begin{gathered}
\frac{1}{n} H_{\mu^{*}}\left(\left(\left(\xi_{-p}^{0}\right)^{*} \vee \mathcal{A}^{*}\right)_{0}^{n} \mid \mathcal{A}^{*}\right) \leq \frac{1}{n} H_{\mu}\left(\left(\xi_{-p}^{0} \vee \mathcal{A}\right)_{0}^{n} \mid \mathcal{A}\right) \\
\rightarrow H_{\mu}\left(\xi_{-\infty}^{0} \vee \mathcal{A} \mid T^{-1}\left(\xi_{-\infty}^{0} \vee \mathcal{A}\right)\right)
\end{gathered}
$$

as $n$ tends to infinity. Therefore $H_{\mu}\left(\mathcal{A} \mid T^{-1} \mathcal{A}\right) \leq H_{\mu}\left(\xi_{-\infty}^{0} \vee \mathcal{A} \mid T^{-1}\left(\xi_{-\infty}^{0} \vee \mathcal{A}\right)\right)$, which means that for any increasing partition, we can find a generating partition with a greater entropy. This proves (10).

Let $\mathcal{A}$ be an increasing and generating $\sigma$-algebra. By Lemma 2.4 we have $(\widehat{\mathcal{A}})^{*}=\widehat{\mathcal{A}}^{*}=\mathcal{B}^{*}$, and as $\mathcal{P}\left(T_{*}\right)$ is included in the remote past of every generating increasing $\sigma$-algebra, we have $\mathcal{P}\left(T_{*}\right) \subset \bigcap_{n>0} T_{*}^{-n} \mathcal{A}^{*}$. Since $\mathcal{P}\left(T_{*}\right) \neq\left\{X^{*}, \emptyset\right\}$, $\mathcal{T}:=\bigcap_{\mathcal{A} \subset T^{-1} \mathcal{A}, \widehat{\mathcal{A}}=\mathcal{B}} \bigcap_{n>0} T^{-n} \mathcal{A}$ possesses at least one set of non-zero finite measure and therefore is a $\sigma$-finite factor. Now, (10) reads as

$$
h_{\mathrm{Pa}}(T)=\sup \left\{H\left(\mathcal{A} \mid T^{-1} \mathcal{A} \vee \mathcal{T}\right), \mathcal{A} \subset T^{-1} \mathcal{A}, \widehat{\mathcal{A}}=\mathcal{B}\right\} .
$$

Thanks to Proposition 7.1. $\sup \left\{H\left(\mathcal{A} \mid T^{-1} \mathcal{A} \vee \mathcal{T}\right), \mathcal{A} \subset T^{-1} \mathcal{A}, \widehat{\mathcal{A}}=\mathcal{B}\right\}$ is the relative Poisson entropy of $T$ with respect to $\mathcal{T}$. But since $h\left(T_{*}\right)=h_{\mathrm{Pa}}(T)<\infty$, we deduce that $h\left(T_{*}, \mathcal{T}^{*}\right)=0$. We can now apply Proposition 11.4.

Corollary 12.2. Assume $(X, \mathcal{B}, \mu, T)$ is of type $\mathbf{I I}_{\infty}$ and $h\left(T_{*}\right)=h_{P a}(T)<\infty$. Assume that $f \in L^{2}(X, \mu)$ is a function such that $\sigma\left(\left\{f \circ T^{n}\right\}_{n \in \mathbb{Z}}\right)=\mathcal{B}$ and $f$ has singular spectral measure. Then $h\left(T_{*}\right)=0$.

Proof. Since $f$ has singular measure (under $T$ ), so has the centered stochastic integral $f^{*}$ (under $T_{*}$ ) (i.e. the image of $f$ under the natural isomorphism between $L^{2}(\mu)$ and the first chaos of $\left.L^{2}\left(\mu^{*}\right)\right)$. Therefore $f^{*}$ is measurable with respect to $\mathcal{P}\left(T_{*}\right)$, so we deduce $\mathcal{P}\left(T_{*}\right) \neq\left\{X^{*}, \emptyset\right\}$. Applying Proposition 12.1, we obtain the 
fact that $\mathcal{P}\left(T_{*}\right)$ is a Poissonian factor. But the smallest Poissonian factor generated by $f^{*}$ is the whole $\sigma$-algebra $\mathcal{B}^{*}$ (as $\mathcal{B}$ is the factor generated by $f$ ), and this ends the proof.

An immediate consequence of Proposition 11.4 is the following:

Corollary 12.3. If two $\mathbf{I I}_{\infty}$-transformations $\left(X, \mathcal{B}_{i}, \mu_{i}, T_{i}\right)$ have zero Poisson entropy for $i=1,2$, then so does any joining of them.

Proof. Let $(X, \mathcal{B}, \nu, T)$ be a joining of $\mu_{1}$ and $\mu_{2}$. By Proposition 11.4. $\left(X^{*}, \mathcal{B}^{*}, \nu^{*}\right.$, $T_{*}$ ) has a Poissonian Pinsker factor, which contains (the pullbacks of) the $\sigma$-algebras $\mathcal{B}_{i}^{*}$ for $i=1,2$. The smallest Poissonian $\sigma$-algebra which contains these is $\left(\mathcal{B}_{1} \vee\right.$ $\left.\mathcal{B}_{2}\right)^{*}=\mathcal{B}^{*}$. Thus $(X, \mathcal{B}, \nu, T)$ is its own Poisson-Pinsker factor.

Except for those cases where the Krengel and Parry entropies coincide with Poisson entropy, we do not know whether the statement corresponding to Corollary 12.3 holds with Krengel or Parry entropy.

We now state a "strong disjointness" result. Recall from [1, page 87, that two systems $(X, \mathcal{B}, \mu, T)$ and $(Y, \mathcal{C}, \nu, S)$ are strongly disjoint if, for any $0<c<\infty$, there is no $T \times S$-invariant $\sigma$-finite measure on $(X \times Y, \mathcal{B} \otimes \mathcal{C})$ whose projections are $\mu$ and $c \nu$, respectively.

Proposition 12.4. Let $(X, \mathcal{B}, \mu, T)$ and $(Y, \mathcal{C}, \nu, S)$ be two ergodic $\mathbf{I I}_{\infty}$-systems. If $(X, \mathcal{B}, \mu, T)$ has a zero Poisson entropy factor and $(Y, \mathcal{C}, \nu, S)$ has not, then they are strongly disjoint.

Proof. By Proposition 11.4 the Pinsker factor of $T_{*}$ is Poissonian. In the proof of Proposition 11.1, we proved that in this situation any factor of $T$ has a $\sigma$-finite intersection with its Poisson-Pinsker factor, and thus has a zero Poisson entropy factor.

Having already used results about relative entropy from 4 in previous sections, we formulate a few other results about Poisson suspensions related to this paper of Danilenko and Rudolph:

Proposition 12.5. Let $(X, \mathcal{B}, \mu, T)$ be an ergodic $\mathbf{I I}_{\infty}$-system with a PoissonPinkser factor $\mathcal{P}$. Then $T$ is relatively $C P E$ (complete positive entropy) and therefore relatively weakly mixing over $\mathcal{P}$.

Proof. It is a consequence of the existence of a relative Pinsker factor with respect to a factor $\mathcal{A}$ (see Definition 1.5 in [4]), which is the maximum factor such that any extension with respect to $\mathcal{A}$ has zero Krengel entropy. Assume $T$ admits $\mathcal{P}(T)$ as a Poisson-Pinsker factor. From Proposition 7.1, relative Poisson and Krengel entropies coincide and thus give the same relative Pinsker factor. But this means that the relative Pinsker factor over $\mathcal{P}(T)$ is $\mathcal{P}(T)$ itself. This proves $T$ is relatively $C P E$ over $\mathcal{P}(T)$. As the maximum distal extension has zero Krengel (and then Poisson) relative entropy, it is also contained in $\mathcal{P}(T)$. Thanks to the infinite Furstenberg decomposition (Proposition 4.2 in [4]), $T$ is relatively weakly mixing over $\mathcal{P}(T)$.

In [4] it is proved that a probability-preserving transformation $S$ is distal if and only if $T \times S$ is a zero entropy extension of $T$ whenever $T$ is a conservative measurepreserving transformation. Translating this result into the Poisson framework yields the following criterion for distality. 
Proposition 12.6. A probability-preserving transformation $S$ is distal if and only if, for any conservative measure-preserving transformation $T$, if the Poisson suspension $T_{*}$ has zero entropy, then so does the Poisson suspension $(T \times S)_{*}$.

We now apply our previous results to a question of Aaronson and Park from [2, regarding the existence of a Krengel-Pinsker factor for quasi-finite transformations. First, we note that the assumptions of Theorem 11.3 hold in particular for quasifinite systems:

Corollary 12.7. Let $T$ be an ergodic quasi-finite system $(X, \mathcal{B}, \mu, T)$. Either it is remotely infinite or there exists a Poisson-Pinsker factor, which is also a Parry and Krengel-Pinsker factor.

Proof. Let $A \in \mathcal{B}$ be a quasi-finite sweep-out set and let $\xi:=\{A, X \backslash A\}$ be the local quasi-finite partition induced by $A$. Observe that the left-hand side of (7) is bounded by the entropy of the return times partition on $A$, thus $H\left(\xi_{-\infty}^{0} \mid T^{-1} \xi_{-\infty}^{0}\right)$ is finite. It follows from (8) and Lemma 11.2 that $\xi_{-\infty}^{0}$ is ED. The assumptions of Theorem 11.3 are thus satisfied.

If there is a Poisson-Pinsker factor $\mathcal{P}$, it is also a Parry-Pinsker factor by Proposition 11.1. To prove it is also a Krengel-Pinsker factor, we have to prove there exists a zero Krengel entropy factor. But if $\mathcal{A}$ is the factor generated by $\xi$, then Poisson and Krengel entropy coincide on this factor and are finite. Moreover, as in the proof of Proposition [1.1, $\mathcal{A} \cap \mathcal{P}$ is $\sigma$-finite, therefore we can consider the extension $\mathcal{A} \cap \mathcal{P}$ to $\mathcal{A}$ where relative Poisson and Krengel entropies coincide thanks to Proposition 7.1. Since $\mathcal{A} \cap \mathcal{P}$ has zero Poisson entropy, it is also the case for Krengel entropy, and we are done.

Corollary 12.7 generalizes a result of from [2] about the existence of a KrengelPinsker factor for a special class of quasi-finite systems called $L L B$. We do not know if the conclusion of this corollary is true without the assumption that $T$ is quasi-finite.

Since we do not know that a factor of a quasi-finite system is itself quasi-finite, we cannot conclude that the remotely-infinite property is inherited by factors in the quasi-finite ergodic case. However, this is the case for $L L B$ systems which are shown to be $L L B$ on any of their factors (see [2] again). As a consequence of Theorem 11.3, we get:

Corollary 12.8. If $T$ is $L L B$ and remotely infinite, then any factor $S$ of $T$ is remotely infinite.

The main open question left at this point, as stated at the beginning, is the following: Are Krengel, Parry and Poisson entropies equal for every conservative measure-preserving transformation?

At this time, we cannot answer even the following questions: Is there an inequality between Poisson entropy and Krengel entropy which holds in general? Are the properties of having zero Poisson entropy and having zero Krengel entropy equivalent?

Related to this is the following question from [4]: Does any conservative transformation have a factor with arbitrarily small Poisson/Krengel entropy? A positive 
answer to Danilenko and Rudolph's question would imply a positive answer to our main question. However we do not even know if there always exists a factor with finite Poisson or Krengel entropy.

\section{ACKNOWLEDGEMENT}

The second author would like to thank his Ph.D. advisor, Professor Jon Aaronson, for his guidance throughout this work. The authors thank A. Danilenko and D. Rudolph for making their paper 4] available prior to its publication, and the referee for a careful reading and useful remarks.

\section{REFERENCES}

[1] J. Aaronson. An introduction to infinite ergodic theory, volume 50 of Mathematical Surveys and Monographs. American Mathematical Society, Providence, RI, 1997. MR:1450400 (99d:28025)

[2] J. Aaronson and K. K. Park. Predictability, entropy and information of infinite transformations. arXiv/0705.2148.

[3] D. Daley and D. Vere-Jones. An introduction to the theory of point processes. Springer-Verlag, New York, 1988. MR950166 (90e:60060)

[4] A. Danilenko and D. Rudolph. Conditional entropy theory in infinite measure and a question of Krengel. Israel J. Math., 72:93-117, 2009. MR 2534241

[5] T. de la Rue. Entropie d'un système dynamique Gaussien : cas d'une action de $Z^{d}$. C. R. Acad. Sci. Paris, 317:191-194, 1993. MR1231420(94c:28022)

[6] N. A. Friedman. Introduction to ergodic theory. Van Nostrand Reinhold Co., New York, 1970. Van Nostrand Reinhold Mathematical Studies, No. 29. MR0435350 (55:8310)

[7] S. Goldstein and J. L. Lebowitz. Ergodic properties of an infinite system of particles moving independently in a periodic field. Comm. Math. Phys., 37:1-18, 1974. MR.0356802 (50:9271)

[8] G. Grabinsky. Poisson process over $\sigma$-finite Markov chains. Pacific J. Math., 111(2):301-315, 1984. MR.734857 (85h:28016)

[9] S. Kalikow. A Poisson random walk is Bernoulli. Comm. Math. Phys., 81(4):495-499, 1981. MR634444(83b:60085)

[10] E. M. Klimko and L. Sucheston. On convergence of information in spaces with infinite invariant measure. Z. Wahrscheinlichkeitstheorie und Verw. Gebiete, 10:226-235, 1968. MR0239874 (39:1231)

[11] U. Krengel. Entropy of conservative transformations. Z. Wahrscheinlichkeitstheorie und Verw. Gebiete, 7:161-181, 1967. MR0218522 (36:1608)

[12] W. Parry. Ergodic and spectral analysis of certain infinite measure preserving transformations. Proc. Amer. Math. Soc., 16:960-966, 1965. MR0181737 (31:5964)

[13] W. Parry. Entropy and generators in ergodic theory. W. A. Benjamin, Inc., New YorkAmsterdam, 1969. MR0262464(41:7071)

[14] W. Parry. Topics in ergodic theory, volume 75 of Cambridge Tracts in Mathematics. Cambridge University Press, Cambridge, 2004. Reprint of the 1981 original. MR2140546 (2005m:37003)

[15] V. Rokhlin. Lectures on the entropy theory of measure-preserving transformations. Russ. Math. Surv., 22:1-52, 1967.

[16] E. Roy. Mesures de Poisson, infinie divisibilité et propriétés ergodiques. Ph.D. thesis, 2005.

[17] E. Roy. Ergodic properties of Poissonian ID processes. Ann. Probab., 35(2):551-576, 2007. MR2308588 (2008d:60054)

[18] E. Roy. Poisson suspensions and infinite ergodic theory. Ergodic Theory Dynam. Systems 29(2):667-683, 2009. MR2486789

[19] J. G. Sinal̆. Ergodic properties of a gas of one-dimensional hard globules with an infinite number of degrees of freedom. Funkcional. Anal. i Priložen., 6(1):41-50, 1972. MR0297288 $(45: 6345)$

[20] K. L. Volkovysskiĭ and J. G. Sină. Ergodic properties of an ideal gas with an infinite number of degrees of freedom. Funkcional. Anal. i Priložen., 5(3):19-21, 1971. MR0289094 (44:6289) 
[21] R. Zweimüller. Poisson suspensions of compactly regenerative transformations. Colloquium Mathematicum, 110:211-225, 2008. MR2353907(2009e:28051)

Laboratoire de Mathématiques Raphä̈l Salem, Université de Rouen, CNRS, Avenue De L'Université, F76801 Saint Étienne du Rouvray, France

E-mail address: Elise.Janvresse@univ-rouen.fr

School of Mathematical Sciences, Tel-Aviv University, Ramat-Aviv, Tel-Aviv 69978, ISRAEL

E-mail address: tomm@post.tau.ac.il

Laboratoire Analyse, Géométrie et Applications, Université Paris 13 Institut Galilée, 99 avenue Jean-Baptiste Clément, F93430 Villetaneuse, France

E-mail address: roy@math.univ-paris13.fr

Laboratoire de Mathématiques Raphaël Salem, Université de Rouen, CNRS, Avenue de l'Université, F76801 Saint Étienne du Rouvray, France

E-mail address: Thierry.de-la-Rue@univ-rouen.fr 